\title{
DISTRIBUCIÓN DEL INGRESO Y CONSUMO DE ALIMENTOS EN MÉXICO*
}

\author{
Nora LUSTIG \\ El Colegio de México
}

\section{INTRODUCCIÓN}

EL OBJETIVo central de este trabajo es obtener una caracterización de la estructura actual del consumo de alimentos, en relación a la distribución familiar del ingreso y estimar la redistribución necesaria para garantizar un nivel de alimentación adecuado, para la población que actualmente ingiere un dieta inferior a la recomendada.

La necesidad de implementar políticas de carácter redistributivo para incrementar el consumo de alimentos de las familias de menores recursos, surge del reconocimiento general que aumentar la oferta de alimentos por sí solo no resuelve los graves problemas de desnutrición de la mayoría de la población, en los países que - como es el caso de México- presentan una extremada concentración del ingreso y de los medios de producción, tanto a nivel familiar como regional. ${ }^{1}$

Se ha dividido este documento en tres partes. En la primera se presenta un diagnóstico de la distribución del ingreso y de la estructura del consumo - sobre todo de los alimentos (Sección II). En segundo lugar, se identifica al grupo que se encuentra en situación crítica desde el punto de vista de la alimentación y se hace una caracterización socioeconómica y demográfica del mismo (Sección III). Por último, se estima la redistri-

\footnotetext{
* Este trabajo es el primer resultado del proyecto "Políticas de consumo y distribución del ingreso", a cargo de la autora y que forma parte del Sistema Alimentario Mexicano, proyecto de la Oficina de Asesores de la Presidencia, bajo la coordinación general de Cassio Luiselli. Se agradece a Raúl Legaspi, investigador asociado del proyecto sobre Políticas, por la colaboración prestada en la elaboración de este documento, y a Antonio Martín del Campo por sus comentarios y recomendaciones. También agradezco a Alicia Contreras, Martha Llanos del Moral y Miguel Santín por su valiosa asistencia.

1 Véase, por ejemplo, H. J. Ramírez, L. Ayluardo, G. Becerra y A. Chávez, "La crisis de alimentos en México" INN-CONACYT-PRUNAL, enero de 1975, p. 17; Naciones Unidas, "El hambre y la malnutrición y una mayor equidad en la distribución de los alimentos", Consejo Mundial de la Alimentación, WFC/1979/6, p. 4.
} 
bución del ingreso necesaria para subsanar la deficiencia alimentaria del grupo en situación crítica (Sección IV).

\section{Distribución DEL INGRESO Y ESTRUCTURA DEL CONSUMO}

\section{Distribución del ingreso}

Existen diversos indicadores para medir la evolución distributiva: la participación de los factores, la trayectoria del salario mínimo real, la distribución personal y familiar del ingreso, etc. Sin embargo, para nuestros fines, la distribución familiar del ingreso es la más adecuada ya que capta el grado de desigualdad dentro de los asalariados y de los "patrones" que permanece encubierto cuando se trabaja a nivel de la participación de los factores; por otra parte, el salario mínimo real también resulta engañoso como indicador cuando sabemos que en 1977, casi un $50 \%$ de las familias percibieron un ingreso inferior al salario mínimo. ${ }^{2}$

La evolución de la distribución familiar del ingreso en (aproximadamente) los últimos veinte años puede observarse en el cuadro $1 .{ }^{3} \mathrm{La}$ aguda desigualdad distributiva que prevalece en México resalta de inmediato si se compara para los tres años analizados el porcentaje de ingreso que corresponde al decil más bajo con el percibido por el 5\% más alto: el primero cercano a $1 \%$ y el segundo alrededor del $25 \%$; el decil más alto, por otra parte, se ha apropiado en promedio de cerca del $40 \%$ del ingreso.

En cuanto a la tendencia, se observa que, tanto el decil más bajo, como el veintil más alto, han visto disminuir su participación. En 1963 el primer decil (732 964 familias) obtuvo $1.69 \%$ del ingreso; mientras que en 1968 (827 765) y 1977 (1 100 000) esta magnitud se redujo a $1.21 \%$ y $1.08 \%$, respectivamente; por otra parte, el último veintil ha disminuido su participación de $28.56 \%$ en 1963 , a $27.15 \%$ y $25.4 \%$ en 1968 y 1977 , respectivamente.

En su conjunto, la distribución del ingreso ha mejorado relativamente para los "grupos medios" (del IV al VII decil); empeorado para los grupos de bajos ingresos (del I al III decil); y se ha deteriorado también para el grupo de altos ingresos (del VIII al X decil). Para las familias comprendidas en el $30 \%$ más bajo la participación ha sido de $7.08 \%$ en 1963 , $6.46 \%$ en 1968 y $6.52 \%$ en 1977 ; las familias entre el IV y VII deciles han aumentado su participación de $22.49 \%$ en 1963 a $24.54 \%$ y $26.41 \%$

\footnotetext{
2 Véase, Coordinación General del Sistema Nacional de Información (1979), Encuesta Nacional de Ingresos y Gastos de los Hogares 1977, Primera Observación, Secretaría de Programación y Presupuesto, agosto, cuadro P2.3, p. 79. El V decil recibe una media de 2693 pesos mensuales, aproximadamente igual al salario vigente en 1977 de 2707 pesos (media simple).

${ }^{3}$ Se han seleccionado estos tres años porque se trata de encuestas comparables.
} 


\section{Cuadro 1}

Distribución del ingreso FAMILIAR EN MÉxico POR DECILEs: 1963,1968 y 1977

\begin{tabular}{llll}
\hline Deciles I & 1963 & 1968 & 1977 \\
\hline I & 1.69 & 1.21 & 1.08 \\
II & 1.97 & 2.21 & 2.21 \\
III & 3.42 & 3.04 & 3.23 \\
IV & & & \\
V & 3.42 & 4.23 & 4.42 \\
VI & 5.14 & 5.07 & 5.73 \\
VII & 6.08 & 6.46 & 7.15 \\
& 7.85 & 8.28 & 9.11 \\
VIII & & & \\
IX & 12.38 & 11.39 & 11.98 \\
Xa & 16.45 & 16.06 & 17.09 \\
Xb & 13.04 & 14.90 & 25.45 \\
& 28.56 & 27.15 & \\
\hline
\end{tabular}

Fuente: Para 1963, 1968 y 1977, E. Hernández Laos y J. Córdova Cha., "Estructura de la distribución del ingreso en México", Comercio Exterior, mayo de 1979, cuadro 4, p. 507.

a Número de familias en cada decil: 1963: 732, 964; 1968: 827, 765; 1977: 1100000.

en 1968 y 1977, respectivamente; en cuanto al tope de la distribución, o sea el $30 \%$ más alto, la participación ha bajado de $70.43 \%$ en 1963 a $69.50 \%$ y $67.06 \%$ en 1968 y 1977, respectivamente. Es decir, con base en esta información, se puede concluir que la evolución de la distribución del ingreso entre 1963 y 1977 muestra una cierta desconcentración en el tope en favor de los grupos llamados medios; sin embargo, los grupos de ingreso más bajos no se han beneficiado de este proceso "desconcentrador" y, por el contrario, su situación (relativa) en 1977 es peor aún que la que prevalecía en 1963 y casi igual a la de 1968.

Para tener una idea más acertada de las condiciones de vida en que se encuentran las familias, es necesario conocer, además de su posición relativa, los niveles absolutos de ingreso que les corresponden. En el cuadro 2 se presenta la distribución de las familias por estrato de ingreso para 1977; allí se puede observar que cerca de un $10 \%$ de las familias en 1977 percibió un ingreso igual o inferior a los 700 pesos mensuales $\mathrm{y}$, que poco más del $50 \%$, a los 3150 pesos mensuales; mientras un porcentaje de familias inferior al $3 \%$ superior, recibió un ingreso mensual mayor o igual a 18000 pesos mensuales. Como la cantidad de 2700 pesos mensuales aproximadamente es igual al salario mínimo prevaleciente en 1977, las cifras anteriores indican que entre el $40 \%$ y $50 \%$ de las familias en 1977, 


\section{Cuadro 2}

\section{DisTRIBUCIÓN DE LAS FAMILIAS POR ESTRATOS DE INGRESO CORRIENTE MENSUAL, 1977}

\begin{tabular}{lcr}
\hline $\begin{array}{c}\text { Estratos de } \\
\text { ingreso }\end{array}$ & $\begin{array}{c}\text { Porcientos } \\
\text { defamilias }\end{array}$ & $\begin{array}{c}\text { Número } \\
\text { de familias }\end{array}$ \\
\hline $0-700$ & 10.45 & 1161931 \\
$700.1-1000$ & 6.30 & 699869 \\
$1000.1-1350$ & 6.70 & 744240 \\
$1350.1-180.0$ & 8.53 & 947669 \\
$1800.1-2400$ & 9.42 & 1047939 \\
$2400.1-3150$ & 10.20 & 1133454 \\
$3150.1-4300$ & 12.60 & 1400719 \\
$4300.1-5725$ & 10.36 & 1151005 \\
$5725.1-7500$ & 8.75 & 9.72235 \\
$7500.1-10.150$ & 7.00 & 777612 \\
$10150.1-13400$ & 4.21 & 469312 \\
$13400.1-18000$ & 2.87 & 319520 \\
Mas de 18000 & 2.61 & 289638 \\
Tota1 & $100: 00$ & 11115143 \\
\hline
\end{tabular}

Fuente: Encuesta Nacional de Ingresos y Gastos de los Hogares de 1977. Primera observación (agosto de 1979), Coordinación General del Sistema Nacional de Información, Secretaría de Programación y Presupuesto, cuadro T2.1, p. 59.

percibieron un ingreso inferior al salario mínimo. ${ }^{4}$ En la medida en que este último refleje el ingreso mínimo necesario para cubrir los requerimientos básicos de consumo, cerca de cinco millones de familias no alcanzaron este mínimo.

\section{Estructura del consumo}

Pasemos ahora a analizar la estructura del consumo y su evolución entre 1968 y 1977. En los cuadros 3 y 4 presentamos la composición porcentual del gasto por grupos socioeconómicos para 1968 y 1977, respectivamente.

Para fines de comparar los dos años, hemos tenido que hacer ciertos ajustes en la información publicada de la encuesta de $1977,{ }^{5}$ porque no

${ }^{4}$ Según la información presentada en la publicación reciente de la Encuesta Nacional de Ingresos y Gastos de los Hogares de 1977 (véase, Coordinación General del Sistema Nacional de Información, agosto de 1979, op. cit.), la proporción de hogares que percibieron un ingreso inferior al salario mínimo fue de un $32 \%$. Esto se debe a un error en la cifra que se usó como salario mínimo para hacer el cálculo; esta cifra fue de 1813 pesos mensuales, en lugar de 2707 pesos. Este error se hizo evidente al computar los ingresos medios por deciles.

5 Coordinación General del Sistema Nacional de Información (1979), op. cit. 


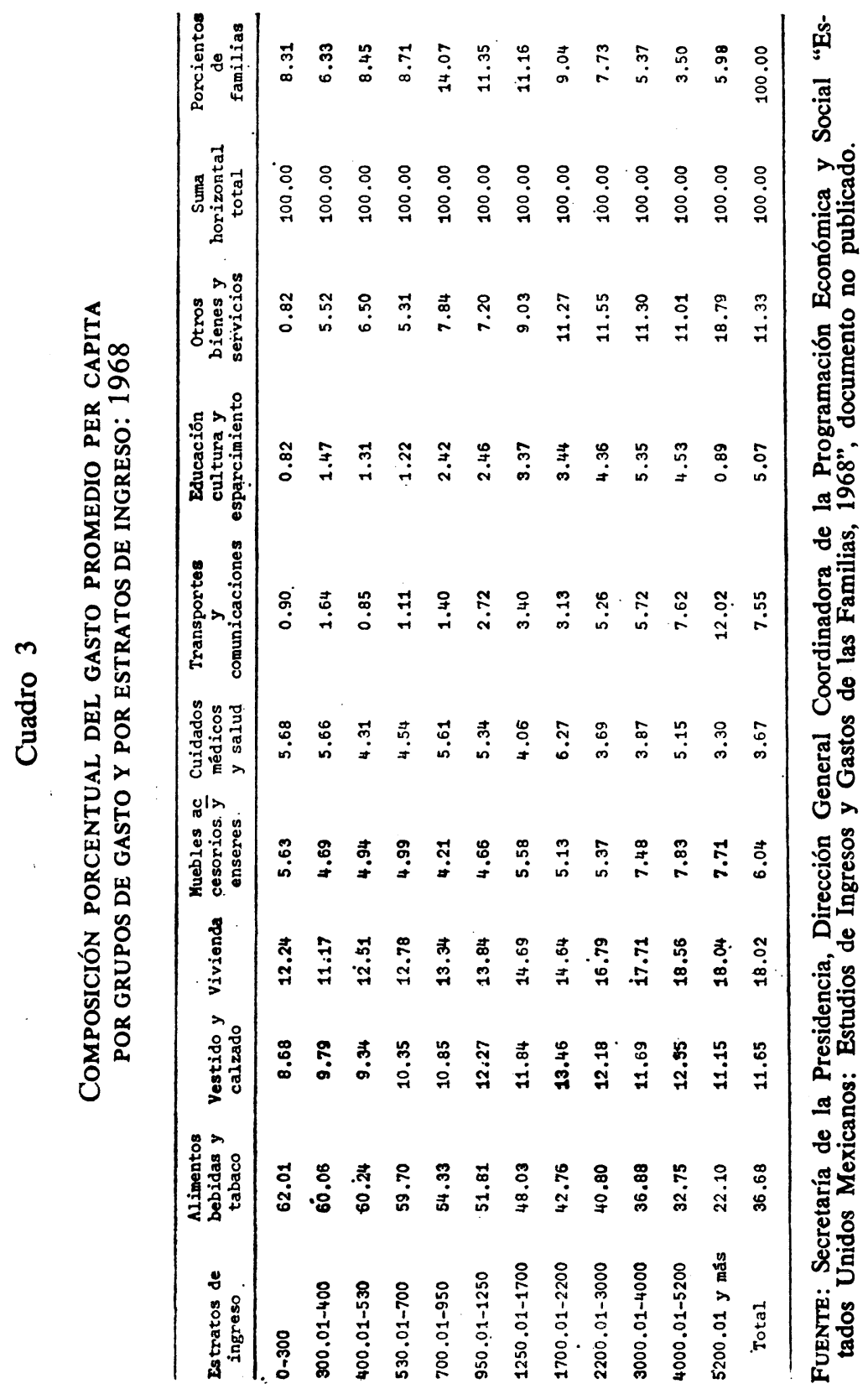


incluye en el rubro de alquileres a la renta imputada, mientras que los datos de 1968 sí lo hacen. ${ }^{6}$ En general, se observa que entre 1968 y 1977 no han habido cambios importantes en la composición porcentual del gasto: destaca un incremento general en el peso de Transportes y Comunicaciones y un ligero descenso en el peso de Alquileres, etc. (Vivienda) que bien puede adjudicarse al mencionado método de ajuste, más que reflejar un hecho real. En el caso del gasto en Alimentos y Bebidas, se puede observar que la proporción ha permanecido casi constante para todos los estratos de ingreso.

En cuanto a las tendencias generales en el interior de las encuestas, es notorio el descenso brusco de la participación del gasto en Alimentos y Bebidas del decil más bajo al veintil más alto. En 1968, por ejemplo, el estrato de 0-300 pesos que corresponde aproximadamente al primer decil (véase el cuadro 3, última columna), destinó $62 \%$ de su gasto total a este rubro, mientras tanto, el estrato de 5201.01 pesos y más (igual al $5.98 \%$ más alto), destinó sólo un $22 \%$ (o sea, desciende alrededor de un $65.5 \%$ ). Para 1977 las cifras análogas fueron de $61.57 \%$ y $20.18 \%$, respectivamente (un descenso de $67.22 \%$ ). ${ }^{7}$ La elevada proporción que gastan en alimentos los sectores de bajos ingresos son el simple reflejo de su pobreza que los obliga a destinar una gran parte de su ingreso al consumo que podría llamarse de subsistencia. ${ }^{3}$ Sin embargo, como se verá más adelante, aun cuando destinan alrededor del $60 \%$ de su gasto monetario corriente al consumo de alimentos, la dieta que ingieren resulta inadecuada e insuficiente desde el punto de vista nutricional.

Una tendencia contraria y también marcada se observa en los rubros de Transporte y Comunicaciones, y Esparcimiento y Educación; en 1968 del I al X deciles éstos aumentan de $0.90 \%$ a $12.02 \%$ y de $0.82 \%$ a $6.89 \%$ (Educación y Esparcimiento están juntos en 1968); en 1977 estos incrementos son de $2.91 \%$ a $18.29 \%$ (Transporte y Comunicaciones), de $0.44 \%$ a $5.48 \%$ (Esparcimiento), y de $0.72 \%$ a $2.93 \%$ (Educación).

Por otra parte, en el cuadro 5 se ha calculado con base en la encuesta de 1977 la participación en el consumo de los distintos bienes, correspondiente a cada decil. Los porcentajes que allí observamos son reflejo de la desigualdad global: es obvio que la concentración del ingreso debe tener su contrapartida en la concentración del consumo. En el caso de Alimentos y Bebidas, por ejemplo, el decil de ingreso más bajo consume cerca del $2 \%$ del total, mientras que el decil más alto consume un $25 \%$.

6 Este ajuste se hizo aplicando a 1977 la misma proporción de ingreso imputado que apareció en 1968, según los deciles aproximadamente correspondientes.

7 Estos porcentajes no son los que aparecen en el cuadro 5, sino los que fueron calculados después de deducir la parte correspondiente a alimentos y bebidas consumidos fuera del hogar.

8 Esto no debe confundirse con la afirmación errónea de algunos autores sobre la ausencia de participación en el consumo de manufacturas por parte de la población de bajos recursos, ya que una proporción importante de los alimentos son manufacturados. 


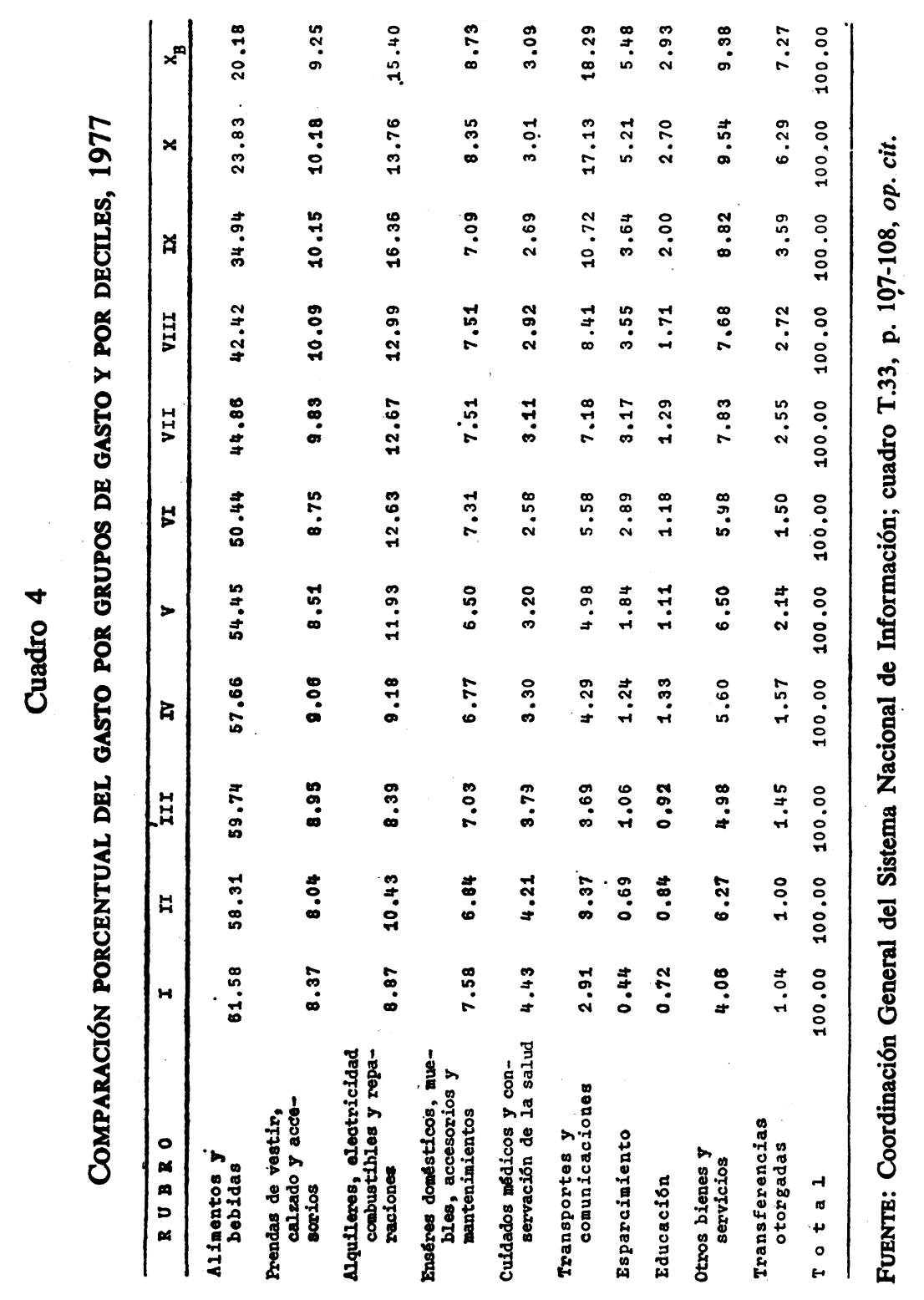




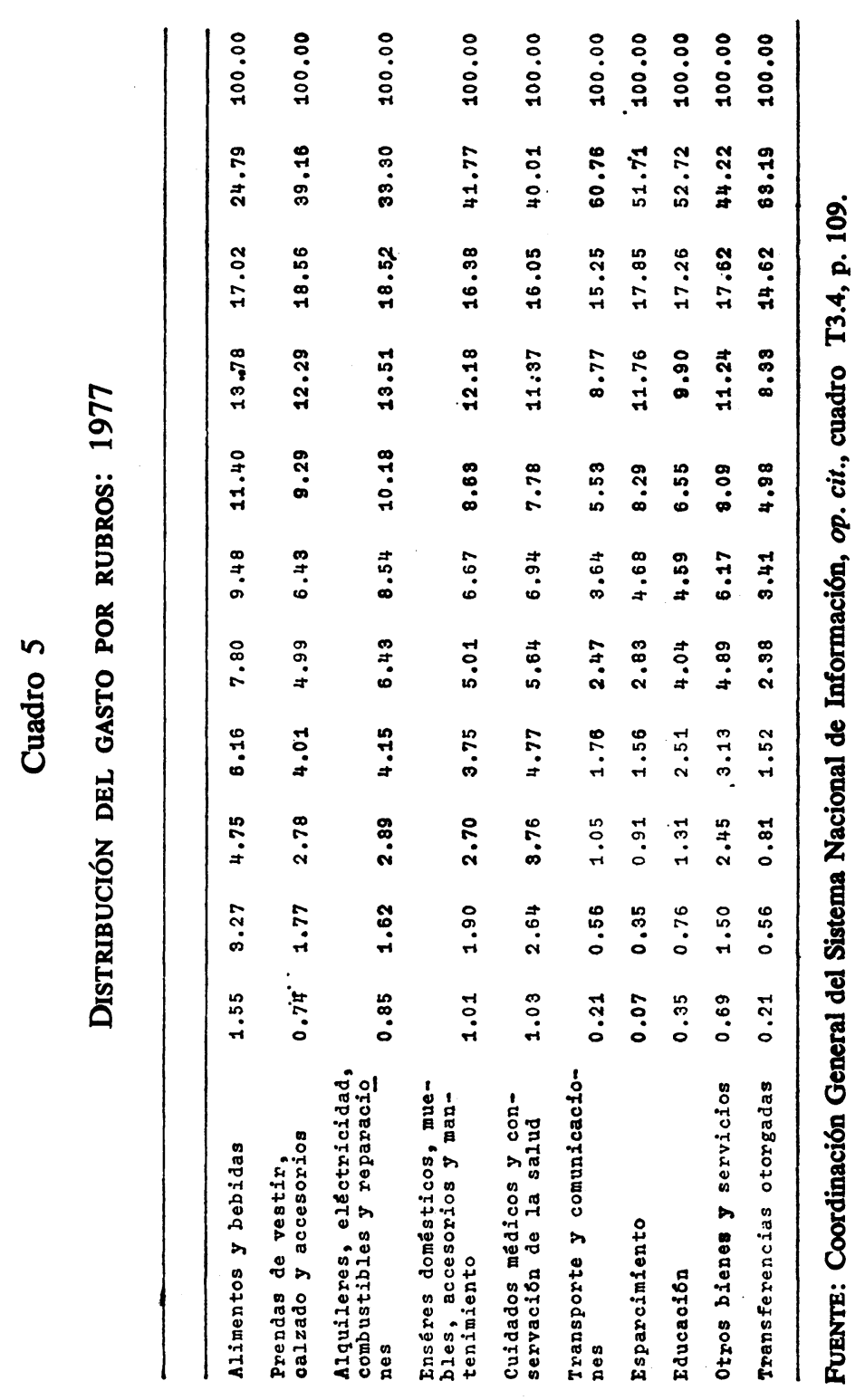


Para Vestimenta y Calzado, la diferencia es entre aproximadamente el $1 \%$ y $39 \%$; para Vivienda, también entre $1 \%$ y $33 \%$; y para Educación, entre $0.3 \%$ y 53 por ciento.

$\mathrm{Si}$ se agrupan los deciles en los tres conjuntos que se definen antes cuando se analizó la evelución distributiva (I-III; IV-VIII; VIII-X), se obtienen los resultados del cuadro 6.

\section{Cuadro 6}

DistribuCión DEL GASTO POR RUBRO POR DECILES AGRUPADOS, 1977

\begin{tabular}{|c|c|c|c|c|}
\hline & I - I I I & IV-VII & VIII -X & Total \\
\hline Alimentos y bebidas & 9.57 & 34.84 & .55 .59 & 100.00 \\
\hline Vestimenta y. cakzado & 5.29 & 24.72 & 70.01 & 100.00 \\
\hline Vivienda & 5.36 & 29.30 & 65.33 & 100.00 \\
\hline Cuidados médicos y salud & $7.4 \dot{3}$ & 25.13 & 67.43 & 100.00 \\
\hline Educacion & 2.42 & 17.69 & 79.88 & 100.00 \\
\hline
\end{tabular}

FUENTE: Cuadro 5.

Es decir, que el grupo de las familias que perciben un ingreso inferior a 1350 pesos mensuales (deciles I-III) ${ }^{9}$ consume únicamente un $10 \%$ de los Alimentos y Bebidas, un $6 \%$ de la Vestimenta y Calzado, un $6 \%$ en Vivienda, un $7 \%$ en Salud y un $2 \%$ en Educación; mientras que el grupo más alto (VIII-X): $56 \%, 70 \%, 65 \%, 67 \%$ y $79 \%$, respectivamente. Este conjunto de datos es un indicador contundente - aunque grueso- de la aguda desigualdad en el acceso a bienes básicos; es un indicador porque en el caso de Salud y Educación, y hasta cierto punto de los otros bienes, parte del consumo está subsidiado ya sea vía la prestación de servicios gratuitos o vía discriminación de precios. ${ }^{10}$ Además aquí únicamente se computa el gasto monetärio, sin incluir el autoconsumo en alimentos que para las familias pobres - sobre todo del sector rural- es muy importante.

La publicación de la Encuesta de Ingresos y Gastos de $1977^{11}$ no trae información sobre el autoconsumo. Sin embargo, se puede obtener una idea aproximada de éste para 1968 que se presenta en el cuadro 7 (se dice aproximada porque las encuestas de este tipo siempre tienden a subestimarlo).

Como era de esperarse, el autoconsumo es importante en el sector rural y a niveles de ingreso relativamente bajos; mientras que en el sector urbano y sobre todo en el metropolitano es casi ínfimo. Para las familias ubicadas en los estratos de gasto entre 0 y 600 pesos mensuales de 1968 en el sector

9 Aproximadamente, ya que este estrato se compone del $31.98 \%$ de los hogares.

10 De aquí se desprende que resulta esencial conocer a qué grupos de la población benefician estos subsidios. Se ha comentado que éstos pueden incluso ser regresivos; o sea, que no benefician a los que tienen menos recursos.

${ }^{11}$ Coordinación General del Sistema Nacional de Información, op. cit. 


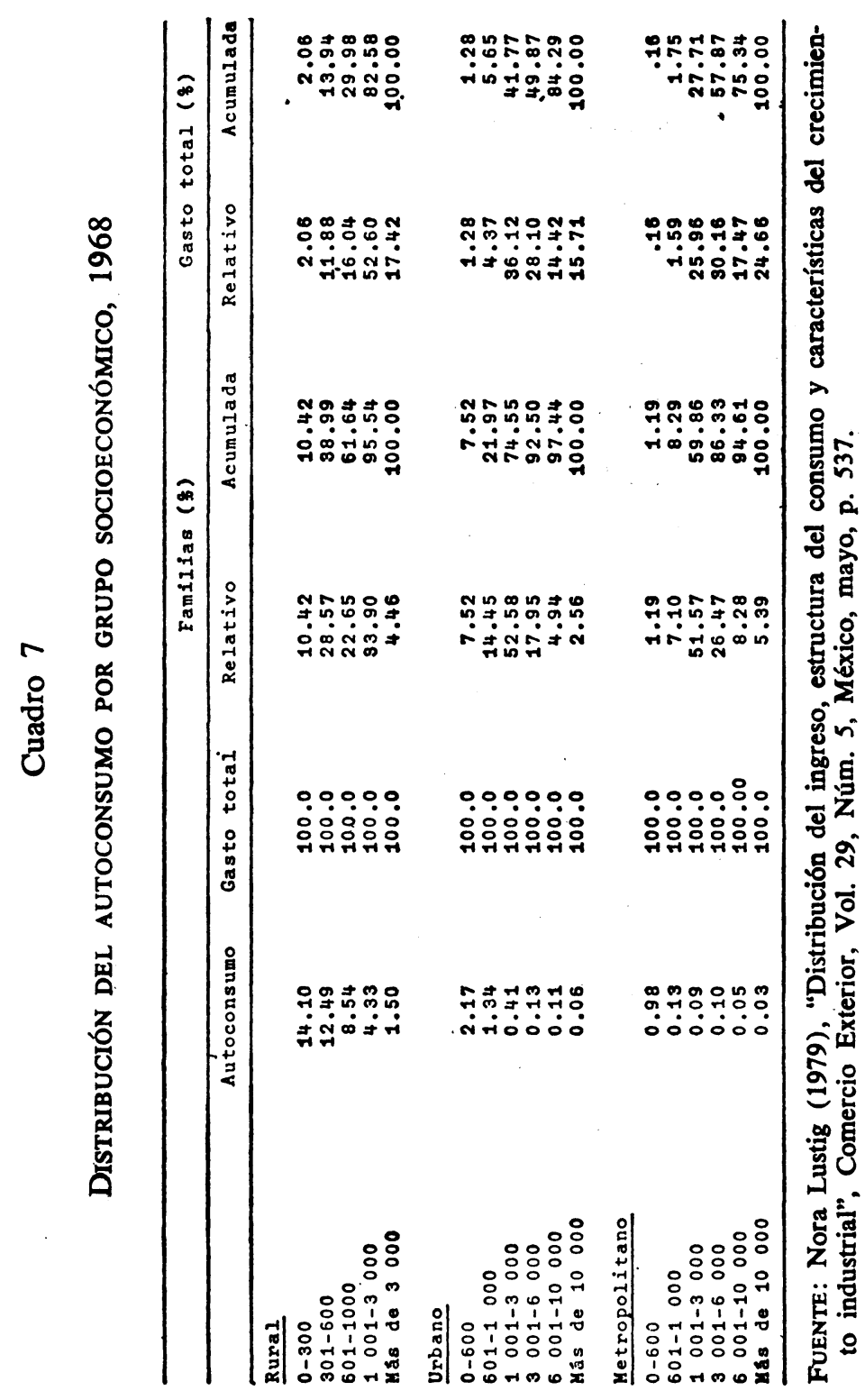


rural $^{12}$ ( $39 \%$ de las familias rurales), el autoconsumo evaluado a precios corrientes resultó en una proporción cercana al $13 \%$ del gasto total. Este mismo grupo de familias destinó alrededor del $60 \%$ del gasto total a Alimentos, Bebidas y Tabaco; o sea, que el autoconsumo convertido a pesos correspondió a aproximadamente un $20 \%$ del gasto en alimentos.

\section{Estructura del consumo de alimentos}

En este apartado se examina la composición del gasto en alimentos en particular, con base en la información de la Encuesta Nacional de Ingresos y Gastos de los Hogares de $1977 .^{13}$

En el cuadro 8 se presenta una lista de bienes y sus rangos para los seis primeros deciles y el total nacional. El rango de cada bien corresponde a un indicador que contempla tanto la frecuencia de familias que consumieron el bien, como la participación del mismo en el gasto total de alimentos. ${ }^{14}$

De acuerdo a esta información, lo que podría denominarse como la canasta básica del grupo de menores ingresos (I-III) está compuesta de maíz en grano, frijol, tomates y, hasta cierto punto, carne de res (que ocupa el octavo lugar en el primer decil, pero el tercero en el II y III). A medida que nos movemos a los deciles superiores, el maíz en grano pierde importancia relativa y la carne de res ocupa el primer lugar. Así se tiene que a nivel nacional, los primeros cuatro productos son: carne de res, pan de dulce, huevos y tortillas de maíz; el frijol y el tomate ocupan el quinto y sexto lugar, respectivamente, y el maíz en grano el doceavo.

En los cuadros 9, 10, 11 y 12 se tiene la participación en el gasto total en alimentos de 50 productos seleccionados por el criterio de frecuencia y peso (constituyen el $96.15 \%$ del gasto en alimentos a nivel nacional), para el total nacional y los deciles I, II y III. Para el primer decil (cuadro 10) se puede observar que un $56 \%$ del gasto en alimentos es absorbido por 7 productos: maíz en grano $(28.75 \%)$; frijol $(7.62 \%)$; café tostado o molido $(4.58 \%)$; carne de res $(4.42 \%)$; manteca de puerco $(4.24 \%)$; aceite vegetal $(3.20 \%)$; y tomates $(2.98 \%)$. En el caso del segundo decil, el $57 \%$ del gasto en alimentos abarca 10 productos y: su distribución es: maíz en grano $(21.54 \%)$; frijol $(6.71 \%)$; carne de res $(5.60 \%)$; manteca de puerco $(3.87 \%)$; leche fresca no pasteurizada $(3.71 \%)$; aceite vegetal (3.16\%); café tostado o molido $(3.11 \%)$; tomates $(3.0 \%)$; huevos $(2.95 \%)$ ) refrescos o sodas $(2.87 \%)$. En el tercer decil (cuadro 12 ), se presentan 11 bienes en el $56 \%$ del gasto total en alimentos, éstos son: maíz en grano (15.64\%); carne de res $(7.18 \%)$; frijol (5.99\%); leche

12 El salario mínimo de ese año era cercano a los 600 pesos mensuales.

13 Coordinación General del Sistema Nacional de Información, op. cit.

14 Véase la Metodología para definir la canasta básica actual, presentada en el subproyecto Núm. 3: Balance de oferta y demanda del Sistema Alimentario Mexicano, Oficina de Asesores de la Presidencia, noviembre de 1979. 
fresca no pasteurizada (4.03\%); tortillas de maíz (4.02\%); manteca de puerco $(3.41 \%)$; aceite vegetal $(3.39 \%)$; refrescos o sodas $(3.39 \%)$; huevos $(3.28 \%)$; tomates $(3.05 \%)$; carne de aves $(2.63 \%)$.

La tendencia que presentan estos tres deciles y que continúa a medida que se sube por la escala de ingresos, es una sustitución relativa del consumo de carbohidratos por proteínas, como lo indica la disminución porcentual de maíz en grano y el aumento de carne de res, huevos, y carne de aves. En el IV decil, maíz en grano sigue ocupando el primer lugar como porcentaje del gasto $(11.18 \%)$, pero en el V y VI deciles esta proporción desciende a $5.45 \%$ y $3.12 \%$, respectivamente; sin embargo, en estos tres deciles, el consumo de tortilla de maíz aumenta su participación a $5.21 \%$ (IV); $6.66 \%$ (V) y $6.84 \%$ (VI); o sea, puede ser que las familias que pertenecen a estos deciles intermedios ya no cuenten con las facilidades para preparar las tortillas en sus casas, por vivir en zonas urbanas. Por

\section{Cuadro 8}

ORDEN DE IMPORTANCIA DE LOS PRINCIPALES ALIMENTOS A NIVEL NACIONAL Y PARA LOS PRIMEROS SEIS DECILES, 1977

\begin{tabular}{|c|c|c|c|c|c|c|c|}
\hline Alimentos & I & II & III & IV & $\mathbf{v}$ & $\nabla \mathbf{I}$ & $\begin{array}{c}\text { Total } \\
\text { naclonel }\end{array}$ \\
\hline Malz en grano & 1 & 1 & 1 & 2 & 9 & 26 & 12 \\
\hline Frijol & 2 & 2 & 2 & 3 & 3 & 4 & 5 \\
\hline Tomates & 3 & 4 & 4 & $\mathbf{5}$ & $\mathbf{5}$ & 6 & 6 \\
\hline Cafe tostado o molido & 4 & 6 & 13 & 9 & 19 & 22 & 22 \\
\hline Especias y aderezos & 5 & 9 & 9 & 13 & 13 & 10 & 11 \\
\hline Manteca de flierco & 6 & 5 & 7 & 14 & 21 & 25 & 25 \\
\hline Azacar morena & 7 & 7 & 14 & 16 & 29 & 24 & 26 \\
\hline Carne de res & 8 & 3 & 3 & 1 & 1 & 1 & 1 \\
\hline Chile & 9 & 17 & 20 & 25 & 22 & 20 & 10 \\
\hline Aceite vegetal & 10 & 10 & 8 & 8 & 8 & 9 & 10 \\
\hline Cebolla & 11 & 15 & 17 & 22 & 23 & 19 & 23 \\
\hline Azácar blanco & 12 & 16 & 24 & 24 & 29 & 33 & 30 \\
\hline Huevos & 13 & 8 & 5 & 4 & 4 & 3 & 3 \\
\hline Carne de fuerco & 14 & 19 & 21 & 19 & 10 & 12 & 13 \\
\hline Arroz & 15 & 14 & 10 & 11 & 17 & 15 & 15 \\
\hline Pan de dulce & 16 & 13 & 16 & 10 & 12 & 13 & 2 \\
\hline Refrescos o sodas & 17 & 11 & 6 & 7 & 6 & 5 & 8 \\
\hline Tortillas de mala & 18 & $2 \dot{2}$ & 12 & 6 & 2 & 2 & 4 \\
\hline Ralces feculentas y derivadas & 19 & 21 & 18 & 20 & 18 & 16 & 17 \\
\hline Lectic fresca no pasteurizada & 20 & 12 & 11 & 12 & 16 & 17 & 20 \\
\hline Pastas para sopa & 21 & 18 & 15 & 15 & 15 & 14 & .18 \\
\hline Pan blanco. & 22 & 20 & 19 & 18 & 11 & 11 & 14 \\
\hline Galletas & 23 & 25 & 25 & 26 & 28 & 31 & 29 \\
\hline Carne de aves & 24 & 23 & 22 & 17 & 7 & 8 & 9 \\
\hline Otros productos de malz & 25 & 24 & 23 & 21 & 24 & $23^{\circ}$ & $: 32$ \\
\hline Platano tabasco & 26 & 26 & 31 & 31 & 31 & 30 & 33 \\
\hline
\end{tabular}


(Continuación)

\begin{tabular}{|c|c|c|c|c|c|c|c|}
\hline Alimentos & 8 & II & III & IV & $\mathbf{v}$ & vI & $\begin{array}{c}\text { Total } \\
\text { nacional }\end{array}$ \\
\hline Marinas de trigo, arrox y preparadas & 27 & 29 & 30 & 29 & 32 & 35 & 94 \\
\hline Queso fresco & 28 & 28 & 28 & 28 & 26 & 21 & 28 \\
\hline Dusces y postres & 29 & 30 & 26 & $\mathbf{s 0}$ & 25 & 18 & 21 \\
\hline Pescado y muriscos eecol y onvasadoe & so & 31 & 25 & 37 & 40 & 42 & 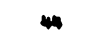 \\
\hline Otros tipos de leche & 31 & 27 & 27 & 27 & 27 & 27 & 27 \\
\hline Verduras y legumbres procesadu & 32 & 37 & 37 & $\boldsymbol{3 0}$ & 99 & 38 & 37 \\
\hline Pescados y marlscos frescos & 83 & 39 & 36 & 36 & 35 & 37 & 35 \\
\hline Leche fresca pasteurizadn & 34 & 93 & 29 & 23 & 24 & 7 & 7 \\
\hline Cafe soluble e instantáneo & 35 & 36 & 34 & 33 & 37 & 92 & 36 \\
\hline otros tipos de carne y carnes procesadas & 36 & 38 & 38 & 34 & 33 & 29 & 16 \\
\hline Otres frutas frescas y frutas procesadas & 37 & * & 32 & 32 & so & 28 & 19 \\
\hline Chocolate, cacao, to & 88 & 32 & 39 & 38 & $\mathbf{3 6}$ & $\boldsymbol{x}$ & 39 \\
\hline Manzana & 99 & 40 & 41 & 42 & 42 & 43 & 42 \\
\hline otros tipos de platano & 40 & 42 & 40 & 41 & 43 & 44 & 47 \\
\hline $\begin{array}{l}\text { Alimentos pmparados y alimento propa- } \\
\text { rados para nifio }\end{array}$ & 41 & 85 & $\boldsymbol{x}$ & 25 & 94 & s4 & 31 \\
\hline Otros tipos de queso & 42 & 45 & 45 & 45 & 45 & 45 & 41 \\
\hline Limon & 43 & 44 & 44 & 40 & $88^{\circ}$ & 98 & 40 \\
\hline Maranja & 4 & 41 & 46 & 46 & 46 & 46 & 45 \\
\hline Aguzcate & 43 & 43 & 43 & 43 & 42 & 40 & 43 \\
\hline Pan caja y otrnes tipos de panes & 46 & 46 & $42 \cdot$ & 44 & 44 & 41 & 38 \\
\hline Mantequilla & 47 & 50 & 48 & 47 & 48 & 48 & 46 \\
\hline Crema & 48 & 48 & 47 & 49 & 47 & 48 & 48 \\
\hline Rechuga & 49 & 49 & so & 30 & 50 & 47 & 50 \\
\hline Zanalicria & 50 & 47 & 49 & 48 & 49 & 49 & 49 \\
\hline
\end{tabular}

Fuente: Secretaría de Programación y Presupuesto; Coordinación General del Sistema Nacional de Información, op. cit.

otro lado, en estos tres deciles - así como en el resto- la carne de res constituye el rubro principal del gasto en alimentos: $7.94 \%$ (IV); $9.19 \%$ (V); y $10.92 \%$ (VI). ${ }^{15}$

A nivel nacional (véase el cuadro 9), el $55 \%$ del gasto en alimentos está destinado a trece productos en el orden siguiente: carne de res (11.32\%); leche fresca pasteurizada (5.42\%); tortillas de maíz $(4.78 \%)$; maíz en grano (4.67\%); carne de aves (4.27\%); huevos $(4.12 \%)$; re-

15 No se dispone de información para los deciles VII al $\mathbf{X}$. 
Cuadro 9

Participación en el gasto de los PRincipales alimentos, SEGÚN SU ORDEN DE IMPORTANCIA: TOTAL NACIONAL, 1977

\begin{tabular}{|c|c|c|c|c|}
\hline & & Gas & $(g) \stackrel{a}{g}$ & Acumulado \\
\hline 1 & Carne de res & 11.32 & (1) & 11.32 \\
\hline 2 & Pan de dulce & 2.55 & (13) & 13.87 \\
\hline 3 & Huevos & 4. 12 & (6) & 13.87 \\
\hline 4 & Tortilla de malz & 4. 78 & ( 3 ) & 22.77 \\
\hline 5 & Frijol & 3.18 & ( 8 ) & 25.53 \\
\hline 6 & Tomates & 2.58 & $(12)$ & 28.53 \\
\hline 7 & Leche fresca pasteurizada & 5.42 & (2) & 33.95 \\
\hline 8 & Refrescos o sodas & 3.54 & ( 7 ) & 37.49 \\
\hline 9 & Carne de aves & 4.27 & ( 5$)$ & 41.76 \\
\hline 10 & Aceite vegetal & 2.77 & (11) & 44.53 \\
\hline 11 & Especias y aderezos & 1.74 & $(20)$ & 46.27 \\
\hline 12 & Maiz en grano & 4.76 & (4) & 50.94 \\
\hline 13 & Carne de puerco & 3.05 & (9) & 53.99 \\
\hline 14 & Pan blanco & 2.06 & $(16)$ & 56.05 \\
\hline 15 & Arroz & 1.30 & $(25)$ & 57.35 \\
\hline 15 & otros tipos de carne y procesados & 2.51 & (14) & 59.86 \\
\hline 17 & Raices feculentas y derivados & 1.22 & $(26)$ & 61.08 \\
\hline 18 & Pastas para sopa & 1.17 & $(27)$ & 62.25 \\
\hline 19 & Otras frutas frescas y procesadas & 2.08 & $(15)$ & 64.33 \\
\hline 20 & Leche fresca no pasteurizada & 2.94 & (10) & 67.17 \\
\hline 21 & Dulces y postres & 1.75 & (19) & 68.92 \\
\hline 22 & Café tostado o molido & 1.58 & $(22)$ & 70.50 \\
\hline 23 & Cebolla & 0.73 & $(37)$ & 71.23 \\
\hline 24 & Chile & 0.89 & ( 33 ) & 72.12 \\
\hline 25 & Manteca de puerco & 1.62 & (21) & 73.74 \\
\hline 26 & Azdcar morena & 0.96 & (31) & 74.70 \\
\hline 27 & Otros tipos de leche & 1.89 & (17) & 76.59 \\
\hline 28 & Queso fresco & 1.37 & $(24)$ & 77.96 \\
\hline 29 & Galletas & 1.23 & $(28)$ & 79.09 \\
\hline 30 & Azacar blanca & 1.01 & $(30)$ & 80.10 \\
\hline 31 & Alimentos preparados y para niño & 1.82 & $(18)$ & 81.92 \\
\hline 32 & Otros productos de maiz & 1.17 & $(27)$ & 83.09 \\
\hline 33 & Platano tabasco & 0.74 & $(36)$ & 83.83 \\
\hline 34 & Harina de trigo, arroz preparado & 1.01 & $(30)$ & 84.84 \\
\hline 35 & Pescados y mariscos frescos & 1.52 & $(23)$ & 86.36 \\
\hline 36 & Café soluble o instantáneo & 1.12 & $(29)$ & 87.48 \\
\hline 37 & Verduras y legumbres procesadas & 0.77 & $(35)$ & 88.25 \\
\hline 38 & Pan caja y otros panes & 0.81 & (34) & 89.06 \\
\hline 39 & Chocolate, cacao, te & 0.71 & $(38)$ & 89.71 \\
\hline 40 & Limón & 0.42 & $(45)$ & 90.19 \\
\hline 41 & Otros tipos de queso & 0.90 & ( 32$)$ & 91.09 \\
\hline 42 & Manzana & 0.63 & (41) & 91.72 \\
\hline 43 & Aguacate & 0.60 & $(42)$ & 92.32 \\
\hline 44 & Pescados y mariscos secos y envasados & 0.70 & $(39)$ & 93.22 \\
\hline 44 & Harina de trigo & 0.64 & $(40)$ & 93.66 \\
\hline 45 & Naranja & 0.54 & $(43)$ & 94.20 \\
\hline 46 & Mantequilla & 0.37 & $(47)$ & 94.57 \\
\hline 47 & Otros platanos & 0.32 & $(48)$ & 94.89 \\
\hline 48 & Crema & 0.39 & $(46)$ & 95.28 \\
\hline 49 & Harina de maiz & 0.45 & $(44)$ & 95.73 \\
\hline 50 & Zanahoria & 0.21 & $(49)$ & 95.94 \\
\hline 51 & Lechuga & 0.21 & $(49)$ & 96.15 \\
\hline
\end{tabular}

FUENTE: Sistema Alimentario Mexicano; Balance de Oferta y Demanda; SUBPROYECTO No. 3.

a Las cifras entre paréntesis indican el orden del producto según su peso que tenga dentro del gasto total en alimentos. 
frescos o sodas $(3.54 \%)$; frijol (3.18\%); carne de puerco $(3.05 \%)$; leche fresca no pasteurizada $(2.84 \%)$; aceite vegetal $(2.77 \%)$; tomates $(2.58 \%)$; y pan de dulce $(2.55 \%)$. Al agregar algunos de estos productos destacan los siguientes renglones: carnes (18.64\%), leche y huevos $(12.38 \%)$ y maíz y derivados $(9.45 \%)$.

En cuanto a las "deformaciones" en el consumo de alimentos éstas se pueden percibir, por un lado, a través de la ubicación relativa de la participación del gasto en refrescos, que ocupa el séptimo lugar a nivel nacional y el decimocuarto, décimo y séptimo para los deciles, I, II y III, respectivamente (véanse los cuadros $9,10,11$ y 12); y, por otro, la tendencia a que su participación aumente aún en los grupos que enfrentan serios problemas de desnutrición: por ejemplo, el gasto en este rubro del total de alimentos del segundo decil es de $2.87 \%$ (cercano al del rubro huevos que es de $2.95 \%$ ); y del tercero de $3.39 \%$ (huevos, $3.28 \%$ ). El consumo de azúcar blanca también representa una "deformación" del consumo por ser más cara y menos nutritiva que el azúcar morena; su consumo representa un $2.06 \%$ del total de alimentos del primer decil, y $1.97 \%$ y $1.51 \%$ del segundo y tercero, respectivamente. También resulta algo alarmante - por sus bajos niveles nutritivos- que a nivel nacional se destine un $1.8 \%$ del gasto en alimentos, a alimentos preparados para niños, y un $0.46 \%$ en el grupo del I al III deciles, dado que es en los primeros años de vida cuando la nutrición adecuada es crucial para la salud física y mental.

\section{DEFINICIÓN Y CARACTERIZACIÓN DEL GRUPo EN SITUACIÓN CRÍTICA}

\section{Distribución del ingreso y nutrición}

La impresión que causan la desigualdad y la concentración de la distribución del ingreso y —en particular — del consumo de alimentos que vimos en las páginas anteriores, se torna más aguda aun cuando se compara el consumo de proteínas y calorías (y otros nutrientes), para los distintos grupos de la población. Entonces se puede observar que el problema de una "mala" distribución no es únicamente relativo sino absoluto, y que existe en la actualidad un número elevado de hogares mexicanos con niveles de desnutrición alarmantes.

En un trabajo hecho por Ana María Flores, basado en los resultados de la encuesta sobre Ingresos y gastos familiares realizado por la Dirección General de Muestreo de la Secretaría de Industria y Comercio durante 1969 y 1970, se encontró que el costo de una dieta familiar adecuada en 1970 era de alrededor de 599 pesos mensuales en la ciudad y 571 pesos en el campo. Como en general pocas familias destinaban algo más que el $65 \%$ de su gasto total a la alimentación, esto llevaría a concluir que todas aquellas familias que en 1970 ganaban menos de mil pesos mensuales corrían el riesgo de padecer desnutrición. A esta categoría de ingreso per- 


\section{Cuadro 10}

Participación en el gasto de los principales alimentos, SEGÚN SU ORDEN DE IMPORTANCIA: DECIL I, 1977

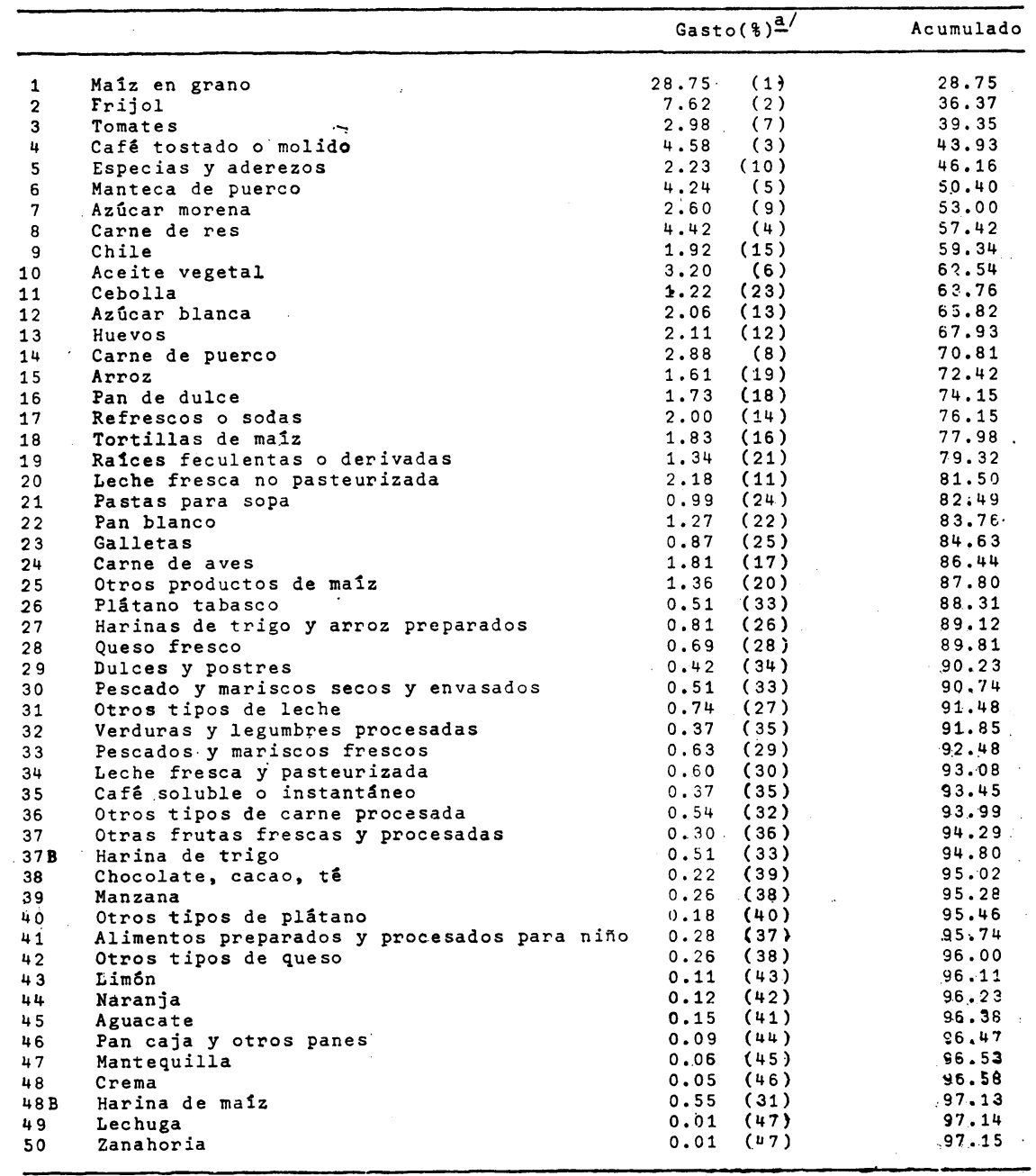

FUENTE: Sistema Alimentario Mexicano; Balance de Oferta y Demanda; SUBPROYECTO No. 3.

a Las cifras entre paréntesis indican el orden del producto según el peso que tenga dentro del gasto total en alimentos. 


\section{Cuadro 11}

\section{Participación en el gasto de los PRincipales alimentos, SEGÚN SU ORDEN DE IMPORTANCIA: DECIL II, 1977}

\begin{tabular}{|c|c|c|c|c|}
\hline \multirow[b]{2}{*}{1} & \multirow[b]{2}{*}{ Malz en grano } & \multicolumn{2}{|c|}{ Gasto $(\%)$ al } & \multirow{2}{*}{$\frac{\text { Acumulada }}{21.54}$} \\
\hline & & 21.54 & (1) & \\
\hline 2 & Frijol & 6.71 & (2) & 28.25 \\
\hline 3 & Carne de res & 5.60 & (3) & 33.85 \\
\hline 4 & Tomate & 3.00 & (8) & 36.85 \\
\hline 5 & Manteca. de puerco & 3.87 & (4) & 40.72 \\
\hline 6 & Cafe tostado o molido & 3.11 & (7) & 43.83 \\
\hline 7 & Azlicar morena & 2.52 & (13) & 46.35 \\
\hline 8 & Huevos & 2.95 & (9) & 49.30 \\
\hline 9 & Especias y aderezos & 1.65 & (1) & 50.95 \\
\hline 10 & Aceite vegetal & 3.16 & (6) & 54.11 \\
\hline 11 & Refrescos o sodas & 2.87 & (10) & 56.98 \\
\hline 12 & Leche fresca no pasteurizada & 3.71 & (5) & 60.69 \\
\hline 13 & Pan de dulce & 2.50 & (14) & 63.19 \\
\hline 14 & Arroz & 1.89 & (18) & 65.08 \\
\hline 15 & Cebolla & 1.14 & (26) & 66.22 \\
\hline 16 & Azlicar blanca & 1.97 & (16) & 68.19 \\
\hline 17 & Chile & 1.33 & $(24)$ & 69.52 \\
\hline 18 & Pastas para sopa & 1.48 & (21) & 71.00 \\
\hline 19 & Carne de puerco & 2.75 & (12) & 73.75 \\
\hline 20 & Pan blanco & 1.91 & (17) & 75.66 \\
\hline 21 & Raices feculentas y derivados & 1.46 & $(22)$ & 77.12 \\
\hline 22 & Tortillas de maiz & 2.80 & (11) & 79.92 \\
\hline 23 & Carne de aves & 2.17 & (15) & 82.09 \\
\hline 24 & Otros productos de maiz & 1.59 & $(20)$ & 83.68 \\
\hline 25 & Galletas & .1 .24 & $(25)$ & 84.92 \\
\hline 26 & Plátano tabasco & 0.72 & $(30)$ & 85.64 \\
\hline 27 & Otros tipos de leche & 1.37 & (23) & 87.01 \\
\hline 28 & Queso fresco & 0.94 & $(28)$ & 87.95 \\
\hline 29 & Harinas de trigo, arroz, preparadas & 0.95 & $(27)$ & 88.30 \\
\hline 30 & Dulces y postres & 0.57 & (37) & 89.47 \\
\hline 31 & Pescados y mariscos secos y envasados & 0.69 & (31) & 90.66 \\
\hline 32 & Chocolate, cacao, te & 0.51 & $(40)$ & 90.57 \\
\hline 33 & Leche fresca pasteurizada & 0.84 & (29) & 91.51 \\
\hline 34 & Otras frutas frescas y procesadas & 0.60 & (35) & 92.11 \\
\hline 35 & Alimentos preparados para niño & 0.56 & (38) & 92.67 \\
\hline 36 & Café soluble o instantáneo & 0.61 & (34) & 93.28 \\
\hline 37 & Verduras y legumbres procesadas & 0.33 & (43) & 93.61 \\
\hline 38 & Otros tipos de carnes y procesados & 0.55 & (39) & 94.16 \\
\hline 39 & Harina de trigo & 0.60 & (35) & 94.76 \\
\hline 40 & Pescados y mariscos frescos & 0.58 & $(36)$ & 94.34 \\
\hline 41 & Harina de masa & 0.64 & (32) & 95.98 \\
\hline 42 & Manzana & 0.36 & (41) & 95.34 \\
\hline 43 & Naranja & 0.35 & $(42)$ & 96.69 \\
\hline 44 & Otros tipos de plátanos & 0.21 & $(45)$ & 96.90 \\
\hline 45 & Aguacate & 0.25 & (44) & 97.15 \\
\hline 46 & Limon & 0.14 & $(47)$ & 97.29 \\
\hline 47 & Otros tipos de queso & 0.63 & (33) & 97.52 \\
\hline 48 & Pan caja y otros tipos de pan & 0.15 & $(46)$ & 97.67 \\
\hline 49 & Zanahoria & 0.07 & (49) & 97.74 \\
\hline 50 & Crema & 0.10 & $(48)$ & 97.84 \\
\hline 51 & Lechuga & 0.05 & $(50)$ & 97.89 \\
\hline 52 & Mantequilla & 0.04 & $(51)$ & 97.93 \\
\hline
\end{tabular}

FUENTE: Sistema Alimentario Mexicano; Balance de Oferta y Demanda; SUBPROYECTO No. 3.

a Las cifras entre paréntesis indican el orden del producto según el peso que tenga dentro del gasto total en alimentos. 
Cuadro 12

Participación en el gasto de los PRINCIPAles alimentos, SEGÚN SU ORDEN DE IMPORTANCIA: DECIL III, 1977

\begin{tabular}{|c|c|c|c|c|}
\hline & & Gasto $(\%)$ & $\underline{a}$ & Acumulado \\
\hline 1. & Maiz en grano & 15.64 & (1) & 15.64 \\
\hline $2^{\circ}$ & Frijol & 5.99 & (3) & 21.63 \\
\hline 3 & Carne de res & 7.18 & (2) & 28.81 \\
\hline 4 & Tomates & 3.05 & $(9)$ & 31.86 \\
\hline 5 & Huevos & 3.28 & (8) & 35.14 \\
\hline 6 & Refrescos o sodas & 3.39 & (7) & 38.56 \\
\hline 7 & Manteca de puerco & 3.41 & (6) & 41.94 \\
\hline 8 & Aceite vegetal & 3.39 & (7) & 45.33 \\
\hline 9 & Especias y aderezos & 1.70 & $(20)$ & 47.03 \\
\hline 10 & Arroz & 2.14 & $(14)$ & 49.17 \\
\hline 11 & Leche fresca no pasteurizada & 4.03 & (4) & 53.20 \\
\hline 12 & Tortillas de maiz & 4.02 & (5) & 57.22 \\
\hline 13 & Café tostado o molido & 2.49 & (11) & 59.71 \\
\hline 14 & Azúcar morena & 1.89 & (18) & 61.60 \\
\hline 15 & Pastas para sopa & 1.62 & $(21)$ & 63.22 \\
\hline 16 & Pan de dulce & 2.33 & (13) & 65.55 \\
\hline 17 & Cebolla & 1.14 & (28) & 66.69 \\
\hline 18 & Ralces feculentas y derivados & 1.61 & $(22)$ & 68.30 \\
\hline 19 & Pan blanco & 2.07 & (15) & 70.37 \\
\hline 20 & Chile & 1.24 & $(27)$ & 71.61 \\
\hline 21 & Carne de puerco & 2.41 & (12) & 74.02 \\
\hline 22 & Carne de aves & 2.63 & $(10)$ & 76.65 \\
\hline 23 & otros productos de maiz & 2.01 & (17) & 78.66 \\
\hline 24 & Azúcar blanca & 1.51 & (23) & 80.17 \\
\hline 25 & Galletas & 1.33 & $(29)$ & 81.50 \\
\hline 26 & Dulces y postres & 1.30 & $(25)$ & 82.80 \\
\hline 27 & otros tipos de leche & 2.06 & $(16)$ & 84.86 \\
\hline 28 & Queso fresco & 1.28 & $(26)$ & 86.14 \\
\hline 29 & Leche fresca pasteurizada & 1.77 & (19) & 87.91 \\
\hline 30 & Harinas de trigo, arroz elaborado & 1.09 & $(29)$ & 89.00 \\
\hline 31 & Plátano tabasco & 0.58 & $(36)$ & 89.58 \\
\hline 32 & Otras frutas frescas y procesadas & 0.60 & (34) & 90.18 \\
\hline 33 & Chocolate, cacao, tê & 0.52 & $(38)$ & 90.70 \\
\hline 34 & Café soluble o instantáneo & 0.69 & (32) & 91.39 \\
\hline 35 & Harina de trigo & 0.69 & $(32)$ & 92.08 \\
\hline 36 & Pescados y mariscos secos y envasados & 0.61 & (33) & 92.69 \\
\hline 37 & Pescados y mariscos frescos & 0.80 & (31) & 93.49 \\
\hline 38 & Verduras y legumbres procesadas & 0.42 & (39) & 93.91 \\
\hline 39 & otros tipos de carne procesada & 0.59 & (35) & 94.50 \\
\hline 40. & Harina de maiz & 0.81 & $(30)$ & 95.31 \\
\hline 41 & Alimentos preparados y procesados para ninos & 0.53 & (37) & 95.84 \\
\hline 42 & otros tipos de platanos & 0.27 & $(44)$ & 96.11 \\
\hline 43 & Manzana & 0.29 & $(42)$ & 96.40 \\
\hline 44 & Pan caja y otros panes & 0.35 & $(40)$ & 96.75 \\
\hline 45 & Aguacate & 0.28 & (43) & 97.03 \\
\hline 46 & Limbn & 0.16 & $(45)$ & 97.19 \\
\hline 47 & Otros tipos de queso & 0.30 & (41) & 97.49 \\
\hline 48 & Naranja & 0.16 & (45) & 97.65 \\
\hline 49 & Crema & 0.13 & $(46)$ & 97.78 \\
\hline 50 & Mantequilla & 0.06 & $(47)$ & 97.84 \\
\hline 51 & Zanahoria & 0.06 & $(47)$ & 97.90 \\
\hline 52 & Lechuga & 0.03 & $(48)$ & 97.9 .3 \\
\hline
\end{tabular}

FUENTE: Sistema Alimentario Mexicano; Balance de Oferta y Demanda; SUBPROYECTO No. 3.

a Las cifras entre paréntesis indican el orden del producto según el peso que tenga dentro del gasto total en alimentos. 
tenecían en 1970 aproximadamente 4.3 millones de familias (o $22.6 \mathrm{mi}$ llones de personas), el $49 \%$ de la población del país. ${ }^{16}$

Por otra parte, Wouter van Ginneken realizó un cálculo de las posibles disparidades entre los niveles nutricionales de la población para 1968 , bajo el supuesto que el promedio diario de alimentos del país alcanzó 2600 calorías y 80 gramos de proteínas per capita en 1970; o sea, algo por encima de los niveles recomendables estimados en esa época por la FAO: 2120 calorías y 45.5 gramos de proteínas diarias; los resultados se presentan en el cuadro 13. ${ }^{17}$ A partir de esta información Van Ginneken encuentra que alrededor del $40 \%$ de las familias que vivían de la agricultura y el $26 \%$ de las familias no agrícolas, no alcanzaron el mínimo recomendado. En total, el número de familias desnutridas resultó en $2.5 \mathrm{mi}$ llones que equivalía a 13.5 millones de personas y representaban el $28 \%$ del país.

En el cuadro 13 también se puede observar la desigualdad existente en el consumo de calorías y proteínas. Para el total del país, el grupo de ingresos más alto compuesto por las familias que ganaban más de 10000 pesos mensuales, consumió casi dos veces más calorías, y 2.68 veces más gramos de proteínas que el grupo de ingresos más bajo, integrado por las familias que percibieron menos de 300 pesos mensuales. ${ }^{18}$

Con el fin de actualizar la información respecto a la ingesta calórica y protéica de la población mexicana, se ha estimado un "perfil nutricional" para 1977 a partir de los datos sobre gasto en alimentos que trae la Encuesta Nacional de Ingresos y Gastos de los Hogares, 1977.19 Los resultados se presentan en el cuadro $14 .^{20}$

Para identificar al grupo de hogares que no cumple con las ingestas recomendadas es necesario determinar, en primer lugar, cuáles deben ser éstas. El Instituto Nacional de la Nutrición, recomienda 2600 calorías y 80 gramos de proteínas diarias per capita; sin embargo, las recomendacio-

16 A. Flores (1973), La magnitud del hambre en México, México, Ed. Impresos Modernos.

17.W. van Ginneken (1974), "Mexican Income Distribution within and between Rural and Urban Areas", World Employment Programme, Working Paper WEP2-23, Ginebra, julio, pp. 62-69.

18 Cuando se compara el porcentaje de la población en este estrato que presenta Van Ginneken (véase cuadro 13) con el que aparece en el cuadro 3, se observa una discrepancia importante: en el cuadro 13 se incluye un $4.3 \%$ de las familias en el estrato de $0-300$ pesos, mientras que en el cuadro 3 un $8.3 \%$. Estas diferencias se deben al factor de expansión de la muestra que se utilizó en cada una de las fuentes $y$, en realidad, es difícil determinar cuál sea la cifra correcta.

19 Este cálculo se hizo gracias a la eficiente labor de programación y cómputo de Raúl Legaspi, investigador asociado del proyecto.

.20 Como se menciona en la nota del cuadro 14, las cifras de calorías y proteínas no son las que se obtuvieron originalmente, sino que fueron ajustadas proporcionalmente en función de la diferencia entre la media nacional arrojada por la encuesta (2 900 calorías y 110 gramos de proteínas) y la media estimada para 1976 por el Instituto Nacional de la Nutrición ( 2600 calorías y 76 gramos de proteínas). 


\section{Cuadro 13}

México: CONSUMO DIARIO DE CALORÍAS Y PROTEÍNAS POR GRUPOS DE INGRESO, 1968

(Dado un promedio nacional de 2600 calorías y 80 gramos de proteínas)

\begin{tabular}{|c|c|c|c|c|c|c|c|c|}
\hline & $\begin{array}{l}\text { Menos de } \\
300 \text { pesos }\end{array}$ & $\begin{array}{l}301 a \\
600\end{array}$ & $\begin{array}{l}6011 \\
1000\end{array}$ & $\begin{array}{l}1001 \text { a } \\
3000\end{array}$ & $\begin{array}{c}3001 a \\
6000\end{array}$ & $\begin{array}{l}6001 a \\
10000\end{array}$ & $\begin{array}{l}10000 \\
0 \text { mass }\end{array}$ & Promedic \\
\hline Calorlas & 1642 & 2071 & 2347 & 2666 & 3272 & 3604 & 4102 & 2600 \\
\hline Protelnas & 51 & 62 & 70 & 82 & 102 & 115 & 134 & 80 \\
\hline * de la poblacion & 4.3 & 14.6 & 19.7 & 41.7 & 14.1 & 3.5 . & 2.1 & \\
\hline
\end{tabular}

FUENTE: Wouter van Ginneken, "Mexican Income Distribution within and between Rural and Urban Areas", World Employment Programme, Working Paper WEP 2-23, Ginebra, julio 1974, p. 67.

nes de la FAO son de 2120 calorías y 45.5 gramos de proteínas diarias. Como es difícil establecer cuál sea el límite correcto, ya que éste depende en gran parte de la composición de la población por edades y por sexo, se ha decidido usar el promedio de ambas recomendaciones que resulta en 2435 calorías y 62.75 gramos de proteínas diarias por persona. En el cuadro 14 se observa que, recién a partir del $\mathrm{V}$ decil, el consumo medio del decil supera estos niveles. O sea que, en 1977 entre un 40 y $50 \%$ de los hogares tuvieron una alimentación insuficiente.

Estos resultados indican que la situación de 1977 fue algo peor que la prevaleciente en 1968: según los datos del cuadro 13, en 1968 poco menos del cuarenta por ciento de los hogares $(38.6 \%)$ no alcanzaron a ingerir los niveles mínimos recomendados de calorías y proteínas; en 1977 este porcentaje es cercano al $45 \%$. Una situación como ésta es una muestra contundente de las contradicciones que ha producido el "modelo" de acumulación mexicano: un crecimiento no sólo desigual en términos relativos, sino acompañado de un empeoramiento (o, en el mejor de los casos, de una constancia) en cuestiones básicas de bienestar como lo es el nivel nutricional de la población.

Por otra parte, es notorio el grado de desigualdad en 1977 en el consumo de calorías y proteínas entre el veintil más alto y el más bajo: el primero ingiere casi dos veces más calorías (1.987) y 3.75 veces más proteínas que el segundo. ${ }^{21}$ Según las fuentes existentes, en 1976 la disponibilidad diaria per capita fue de 2605 calorías y 75.8 gramos de proteínas. Estas cifras son un indicador de que la desnutrición prevaleciente

21 Hasta donde son comparables las encuestas, la distancia entre los consumos de los más ricos y los más pobres también parecen haberse incrementado en 1977 en comparación con 1968. 


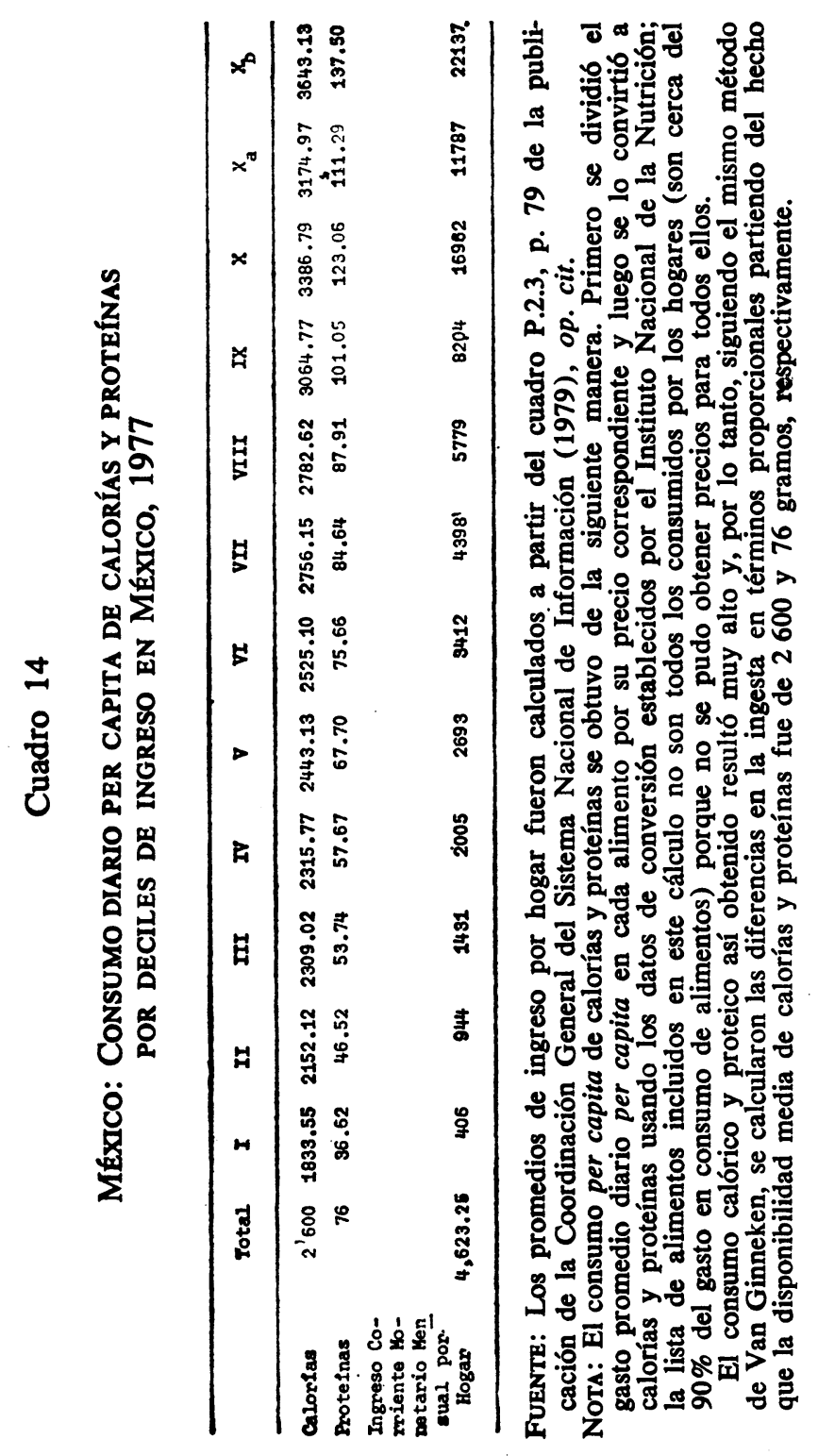


en los grupos de bajos ingresos se debe más a la distribución desigual de los alimentos, que a su escasez; y la distribución desigual de los alimentos es - a su vez - un reflejo de la desigualdad y concentración de la distribución de los ingresos, de los medios de producción y del poder en general, que caracteriza a la sociedad mexicana. ${ }^{22}$

$\mathrm{El}$ análisis precedente permite definir con cierta precisión al grupo que se encuentra en situación crítica, o sea que consume una cantidad de proteínas y calorías inferior a la recomendada. ${ }^{23}$ Dicho grupo está compuesto por los hogares ubicados entre el I y el $\mathrm{V}$ decil y que, de manera coincidente han recibido un ingreso monetario inferior o igual al salario mínimo medio vigente en $1977 .{ }^{24}$

En la siguiente sección se presenta una caracterización del grupo que se encuentra en situación crítica, en base a una serie de variables socioeconómicas y demográficas.

\section{Características socioeconómicas y demográficas de los hogares}

En el cuadro 15 se presenta la composición porcentual de ciertas características de los hogares para los dos grupos que hemos identificado anteriormente: del I al V decil, el grupo en situación crítica; y del VI al X. Los resultados de este cuadro indican una carga de dependencia mayor para el primer grupo: $44.75 \%$ de las personas están en el rango de menos de 12 y más de 64 años de edad; en el segundo grupo este porcentaje es de 36.98 por ciento.

Los datos sobre educación reflejan la desigualdad global. El porcentaje de analfabetismo es algo mayor al $40 \%$ en los jefes del primer grupo y casi un $90 \%$ de los hogares en este grupo tienen un jefe sin instrucción o con primaria incompleta. Por otra parte, este último porcentaje en el segundo grupo es de cerca del $50 \%$ lo cual pareciera indicar que la educación del jefe no es una condición necesaria para lograr niveles de ingreso más altos. Sin embargo, el hecho que en el segundo grupo el $9.24 \%$ de los jefes tenían estudios universitarios completos e incompletos o de posgrado, mientras que en el otro grupo sólo el $0.25 \%$ logra este nivel, parece indicar que la educación del jefe es una condición suficiente para que el ingreso del hogar sea más alto.

22 Más aún, la "crisis" alimentaria o sea, la escasez que enfrenta actualmente México, es - hasta cierto punto- también consecuencia de estas desigualdades.

23 Aquí se ha definido como situación crítica cuando el consumo de calorías y proteínas es menor al recomendado, independientemente de la diferencia cuantitavita. Esta terminología no corresponde a la usada por el Instituto Nacional de la Nutrición, para el cual la situación crítica se refiere a aquellos casos de niveles altísimos de desnutrición.

24 Es interesante observar que el ingreso medio del V decil es de 2693 pesos, prácticamente igual al salario mínimo promedio de 2707 pesos. Es decir que, según nuestros resultados, un ingreso mensual del hogar, equivalente a un salario mínimo parece ser suficiente para consumir una dieta adecuada por lo menos en cuanto a calorías y proteínas se refiere. 
Cuadro 15

COMPOSICIÓN PORCENTUAL DE LAS CARACTERÍSTICAS SOCIOECONÓMICA Y DEMOGRÁFICAS DE LOS HOGARES POR GRUPOS Y DECILES, 1977

\begin{tabular}{|c|c|c|c|}
\hline & Total & $I-V$ & VI $-X$ \\
\hline MUMERO DE HOGARES & 100.00 & 100.00 & 100.00 \\
\hline Numero de Hogares con Jefe & 99.81 & 99.92 & 99.70 \\
\hline MUMERO DE.PERSONAS: & 100.00 & 100.00 & 100.00 \\
\hline Namero de Personas 12 y 64 anos & 40.56 & 44.75 & 36.98 \\
\hline NUMERO DE OCUPADOS & 28.94 & 28.24 & 29.56 \\
\hline INSTRUCCION DEL JEFE DEL HOGAR & 100.00 & 100.00. & 100.00 \\
\hline $\begin{array}{l}\text { Sin Instruccion } \\
\text { Con Primaria Incompleta } \\
\text { Primaria Completa } \\
\text { Instruccion Media y Media Superior } \\
\text { Completa e Incompleta } \\
\text { Con Estudios Universitarios Completos } \\
\text { e Incompletos y Posgrados } \\
\text { No Especificado }\end{array}$ & $\begin{array}{r}27.68 \\
40.04 \\
15.23 \\
11.51 \\
4.74 \\
.80\end{array}$ & $\begin{array}{r}41.68 \\
45.78 \\
8.33 \\
3.00 \\
.25 \\
.96\end{array}$ & $\begin{array}{r}13.65 \\
34.27 \\
22.15 \\
20.04 \\
9.24 \\
.65\end{array}$ \\
\hline TAMANO DEL MUNICIPIO & 100.00 & 100.00 & 100.00 \\
\hline $\begin{array}{l}10,000 \text { Habitantes } \\
\text { De } 10,001 \text { a } 100,000 \text { Habltantes } \\
\text { Mas de } 100,000 \text { Habitantes } \\
\text { Areas Metropolitanas }\end{array}$ & $\begin{array}{l}12.47 \\
37.79 \\
23.07 \\
26.67\end{array}$ & $\begin{array}{r}20.95 \\
56.02 \\
14.50 \\
8.53\end{array}$ & $\begin{array}{r}3.98 \\
19.57 \\
31.64 \\
44.81\end{array}$ \\
\hline TAMANO DEL HOGAR & 100.00 & 100.00 & 100.00 \\
\hline $\begin{array}{l}6 \text { Miembros o Menos } \\
\text { Mas de } 6 \text { Miembros }\end{array}$ & $\begin{array}{l}67.12 \\
32.88\end{array}$ & $\begin{array}{l}71.60 \\
28.40\end{array}$ & $\begin{array}{l}62.68 \\
37.32\end{array}$ \\
\hline FUENTES DE INGRESO & 100.00 & 100.00 & 100.00 \\
\hline $\begin{array}{l}\text { Remuneraciones al Trabajo } \\
\text { Renta Empresarial } \\
\text { Renta Recibida de la Propiedad } \\
\text { Otros Ingresos }\end{array}$ & $\begin{array}{r}70.10 \\
22.37 \\
.96 \\
6.57\end{array}$ & $\begin{array}{r}60.05 \\
31.14 \\
.21 \\
8.60\end{array}$ & $\begin{array}{r}72.03 \\
20.68 \\
1.11 \\
6.18\end{array}$ \\
\hline
\end{tabular}

FUENTE: Secretaría de Programación y Presupuesto. Coordinación General del Sistema Nacional de Información, op. cit. Cuadro T2.3, p. 61 y cuadro T1.3, p. 25.

La distribución de los hogares por tamaño del municipio refleja, aunque de manera deficiente, su distribución urbano-rural. ${ }^{25}$ En este aspecto se observa que un $77 \%$ de los hogares del primer grupo se encuentran en municipios con menos de 100000 habitantes, en contraste con un $24 \%$ de los hogares del grupo más alto. Por otra parte, sólo un $9 \%$ del primer grupo se ubica en áreas metropolitanas, mientras que del segundo grupo lo hacen el $45 \%$ de los hogares. Estos resultados parecen indicar que una alta proporción de los hogares dentro de nuestro grupo objetivo (I-V) se encuentra en zonas rurales.

La participación relativa de las fuentes de ingreso presentadas en el cuadro 15 puede a primera vista resultar sorprendente: por ejemplo, la renta empresarial del primer grupo resulta ser poco más del $30 \%$ del ingreso total percibido por este grupo, mientras que la remuneración al

25 Es deficiente porque hay municipios grandes que abarcan localidades rurales y municipios pequeños que pueden estar insertos en zonas urbanas. 
trabajo es de $60 \%$; por otro lado, los porcentajes análogos son de $72 \%$ y $21 \%$ para el segundo grupo.

$\mathrm{Al}$ respecto hay dos elementos que se deben tomar en cuenta. Por un lado, en el segundo grupo la renta empresarial y la renta recibida de la propiedad probablemente estén subestimadas, como suele suceder en este tipo de encuestas. En segundo lugar, la relativamente alta participación de la renta empresarial del primer grupo no debe interpretarse como una proliferación de "capitalistas" pobres, sino que debe verse como otro reflejo más de la marginación que ha acompañado al proceso de acumulación en México. En este primer grupo se encuentra un elevado número de hogares cuyos miembros no han podido incorporarse a la fuerza de trabajo industrial y que en su mayoría subsisten mediante la explotación de minifundios a nivel de "subsistencia" o "infrasubsistencia" o de las "ganancias" generadas por pequeños comercios familiares.

Si unimos estos resultados con los obtenidos para la distribución de las familias por tamaño del municipio, parece reforzarse la conclusión de que el primer grupo está compuesto en una alta proporción por hogares que pertenecen al sector rural y cuya actividad principal es la explotación agrícola en muy pequeña escala (minifundios); o sea, son fundamentalmente familias campesinas.

Nuestras conclusiones se robustecen si se consideran, por ejemplo, los tres primeros deciles del primer grupo y los tres últimos del segundo, y se comparan sus características socioeconómicas entre sí. En el grupo compuesto por los deciles I-III, el porcentaje de hogares que habitan en municipios con menos de 100000 habitantes es de $87 \%$ y en áreas metropolitanas de $4.5 \%$; en cambio, en aquel compuesto por los deciles VIII-X, estos porcentajes son del $18 \%$ y $49 \%$, respectivamente. Por otra parte, según las fuentes del ingreso el grupo I-III recibe $41 \%$ del suyo en la forma de renta empresarial y el $48 \%$ como remuneraciones al trabajo; en contraste, el grupo VIII-X lo hace en las proporciones de $21 \%$ y $72 \%$, respectivamente.

Estos resultados, combinados con los anteriores, llevan a concluir que a medida que nos acerquemos a los hogares de ingreso más bajo en el primer grupo del I al V decil, es más alta la probabilidad que nos encontremos con familias campesinas. Mientras que los hogares que perciben ingresos más altos dentro de este grupo, probablemente sean familias pobres urbanas que viven primordialmente de la venta irregular de la fuerza de trabajo de sus miembros y que perciben salarios muy bajos.

\section{REDISTRIBUCIÓN DEL INGRESO Y CONSUMO DE ALIMENTOS}

\section{Distribución del ingreso y dinámica del consumo}

En esta sección se analizan las tendencias en la designación del gasto que presentan distintos grupos socioeconómicos, ante cambios en sus ingresos. 
Cuadro

RESUltados EMPÍRICOS DE LAS FUN

\begin{tabular}{|c|c|c|c|c|c|c|c|c|}
\hline \multirow{2}{*}{ Estrato } & \multicolumn{3}{|c|}{ Autoconsumo } & \multicolumn{3}{|c|}{ Primario } & \multicolumn{2}{|r|}{ Tradicion } \\
\hline & $R^{2}$ & $\mathbf{b}$ & c & $R^{2}$ &.$b$ & c & $\mathrm{R}^{2}$ & b \\
\hline $\begin{array}{l}\text { SECTOR RURAT } \\
0 \text { (148) } 300\end{array}$ & .01 & $\begin{array}{c}-.31584 \cdot \\
(.949)\end{array}$ & $\begin{array}{l}.38192 \\
(.755)\end{array}$ & .13. & $\begin{array}{l}.03114 \\
(.082)\end{array}$ & $\begin{array}{c}.67564 \\
(21.090)\end{array}$ & .57 & $\begin{array}{l}.07642 \\
(1.803)\end{array}$ \\
\hline${ }^{301}(384)^{600}$. & .01 & $\begin{array}{l}.45279 \\
(3.042)\end{array}$ & $\begin{array}{l}.64715 \\
(1.364)\end{array}$ & .12 & $\begin{array}{l}.15699 \\
(7.967)\end{array}$ & $\begin{array}{c}.75686 \\
(40.629)\end{array}$ & .17 & $\begin{array}{r}-.18610 \\
(9.092)\end{array}$ \\
\hline${ }_{(392)}^{601}$ & .06 & $\begin{array}{c}1.15129 . \\
(22.373)\end{array}$ & $\begin{array}{l}.18678 \\
(.062)\end{array}$ & .04 & $\begin{array}{l}.09391 \\
(3.352)\end{array}$ & $\begin{array}{c}.58627 \\
(13.667)\end{array}$ & .11 & $\begin{array}{c}-.11925 \\
(5.576)\end{array}$ \\
\hline $\begin{array}{c}1001{ }_{(608)}^{3000} \\
.\end{array}$ & .05 & $\begin{array}{r}1.12574 \\
(.31 .916)\end{array}$ & $\begin{array}{r}-.57719 \\
(3.909)\end{array}$ & .17 & $\begin{array}{c}.14064 \\
(11.586)\end{array}$ & $\begin{array}{c}.63292 \\
(109.317)\end{array}$ & .36 & $\begin{array}{c}.13124 \\
(23.555)\end{array}$ \\
\hline $\begin{array}{c}\text { más de } 30,000 \\
(92)^{\circ}\end{array}$ & .16 & $\begin{array}{r}1.96852 \\
(15.195)\end{array}$ & $\begin{array}{r}-1.11331 \\
(1.738)\end{array}$ & .25 & $\begin{array}{l}.03187 \\
(.105)\end{array}$ & $\begin{array}{c}.87938 \\
(28.608)\end{array}$ & .27 & $\begin{array}{l}.10545 \\
(1.947)\end{array}$ \\
\hline $\begin{array}{l}\text { SECTOR URBANO } \\
0 \quad 1000 \\
(680)\end{array}$ & .09 & $\begin{array}{c}.77339 \\
(31.995)\end{array}$ & $\begin{array}{l}-1.23537 \\
(36.942)\end{array}$ & $: 31$ & $\begin{array}{c}.13503 \\
(22.040)\end{array}$ & $\begin{array}{c}.69267 \\
(262.473)\end{array}$ & .59 & $\begin{array}{c}.09658 \\
(22.037)\end{array}$ \\
\hline $\begin{array}{c}1001 \quad 3000 \\
(20978)\end{array}$ & .01 & $\begin{array}{l}.11057 \\
(4.212)\end{array}$ & $\begin{array}{r}.26300 \\
(9.463)\end{array}$ & .27 & $\begin{array}{c}.16120 \\
(87.250)\end{array}$ & $\begin{array}{c}.68973 \\
(634.251)\end{array}$ & .46 & $\begin{array}{c}.16004 \\
(169.561)\end{array}$ \\
\hline $8001 \frac{6000}{(844)}$ & .004 & $\begin{array}{l}.10197 \\
(2.041)\end{array}$ & $\begin{array}{l}-.23973 \\
(1.678)\end{array}$ & .16 & $\begin{array}{c}.21008 \\
(52.404)\end{array}$ & $\begin{array}{c}79016 \\
(110.244)\end{array}$ & .29 & $\begin{array}{c}.29608 \\
(225.991)\end{array}$ \\
\hline $60_{(251)}^{001} 10000$ & .01 & $\begin{array}{l}.24327 \\
(2.813)\end{array}$ & $\begin{array}{r}-.04892 \\
(.010)\end{array}$ & .14 & $\begin{array}{l}.14424 \\
(6.409)\end{array}$ & $\begin{array}{l}1.07216 \\
(31.517)\end{array}$ & .22 & $\begin{array}{l}.28457 \\
(54.244)\end{array}$ \\
\hline $\begin{array}{l}\text { más de }{ }^{-10} 000 \\
(125)\end{array}$ & 0.2 & $\begin{array}{l}.30763 \\
(1.739)\end{array}$ & $\begin{array}{l}-.33095 \\
(1.005)\end{array}$ & .29 & $\begin{array}{l}.27664 \\
(9.849)\end{array}$ & $\cdot \begin{array}{c}.73074 \\
(34.318)\end{array}$ & .39 & $\begin{array}{c}.44965 \\
(46.458)\end{array}$ \\
\hline SECTOR METROPOI & TANO & & & & & & & \\
\hline $\begin{array}{c}0 \\
(214)\end{array}$ & .03 & $\begin{array}{l}.43295 \\
(6.804)\end{array}$ & $\begin{array}{r}-.38337 \\
(1.167)\end{array}$ & .19 & $\begin{array}{l}.07484 \\
(2.286)\end{array}$ & $\begin{array}{c}.70932 \\
(44.921)\end{array}$ & .41 & $\begin{array}{l}.07013 \\
(4.430)\end{array}$ \\
\hline $\begin{array}{c}10013000 \\
(1141)\end{array}$ & .0001 & - & $\begin{array}{l}.02367 \\
(.116)\end{array}$ & .25 & $\begin{array}{c}.16699 \\
(49.309)\end{array}$ & $\begin{array}{c}.66823 \\
(309.118)\end{array}$ & .48 & $\begin{array}{c}.18255 \\
(123.045)\end{array}$ \\
\hline $\begin{array}{c}3001{ }_{(539)}^{6000} \\
{ }_{(53)}\end{array}$ & .001 & $\begin{array}{l}.06736 \\
(.769)\end{array}$ & $=$ & .14 & $\begin{array}{r}.20361^{\circ} \\
(29.732)\end{array}$ & $\begin{array}{c}.71868 \\
(57.537)\end{array}$ & .34 & $\begin{array}{c}.32079 \\
(184.097)\end{array}$ \\
\hline 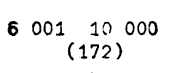 & .02 & $\begin{array}{l}, 2.5833 \\
(3.335)\end{array}$ & $\begin{array}{l}.21430 \\
(.206)\end{array}$ & .10 & $\begin{array}{l}.07639 \\
(1.060)\end{array}$ & $\begin{array}{l}1.06019 \\
(18.301)\end{array}$ & .20 & $\begin{array}{c}.29667 \\
(40.890)\end{array}$ \\
\hline $\begin{array}{l}\text { más de } 10000 \\
\text { (105) }\end{array}$ & .03 & $\begin{array}{l}.33476 \\
(2.877)\end{array}$ & $\begin{array}{r}-.07432 \\
(.069)\end{array}$ & .27 & $\begin{array}{l}.26043 \\
(8.498)\end{array}$ & $\begin{array}{c}.63507 \\
(24.533)\end{array}$ & .43 & $\begin{array}{c}.41081 \\
(48.256)\end{array}$ \\
\hline
\end{tabular}

NorAs: $\mathrm{b}=$ elasticidad-tamaño familiar. $\mathrm{c}=$ elasticidad-ingreso. La cifra ciones en el resto es el valor de la t.

FuENTE: Lustig; N.; Distribución del Ingreso, Estructura del Consumo y 5, México, mayo de 1979, pp. 535-543. 
CIONES DOBLE-LOGARÍTMICAS ESTIMADAS

\begin{tabular}{|c|c|c|c|c|c|c|c|c|c|}
\hline \multirow{2}{*}{$\frac{I}{c}$} & \multicolumn{3}{|c|}{ Iradicional II } & \multicolumn{3}{|c|}{ Hoderno } & \multicolumn{3}{|c|}{ Servicios } \\
\hline & $\mathrm{R}^{2}$ & $b$ & c & $\mathrm{R}^{2}$ & $\mathbf{b}$ & c & $R^{2}$ & $\mathbf{b}$ & c \\
\hline $\begin{array}{l}1.03733 \\
(80.687)\end{array}$ & .39 & & $\begin{array}{r}1.94010 \\
(93.163)\end{array}$ & .04 & $\begin{array}{l}.07028 \\
(.182)\end{array}$ & $\begin{array}{l}50478 \\
(5.101)\end{array}$ & .07 & $\begin{array}{l}-.33916 \\
(4.116)\end{array}$ & $\begin{array}{r}.68104 \\
(.028)\end{array}$ \\
\hline $\begin{array}{l}.10634 \\
.70 .505)\end{array}$ & .08 & $\begin{array}{l}-.14064 \\
(1.750)\end{array}$ & $\cdot \begin{array}{l}1.29751 \\
(32.673)\end{array}$ & .03 & $\begin{array}{l}.17119 \\
(1.558)\end{array}$ & $\begin{array}{l}.93250 \\
(10.146)\end{array}$ & .11 & $\begin{array}{l}-.40753 \\
(15.579)\end{array}$ & $\begin{array}{c}1.29093 \\
(34.300)\end{array}$ \\
\hline $\begin{array}{l}(.00421 \\
.41 .368)\end{array}$ & .06 & $\begin{array}{r}-.05719 \\
(.598)\end{array}$ & $\begin{array}{r}1.07761 \\
(22.219)\end{array}$ & .01 & $\begin{array}{l}.17559 \\
(1.291)\end{array}$ & $\begin{array}{l}75410 \\
(2.490)\end{array}$ & .18 & $\begin{array}{l}-.52158 \\
(32.628)\end{array}$ & $\begin{array}{l}2.11600 \\
(56.174)\end{array}$ \\
\hline $\begin{array}{l}.68378 \\
297.888)\end{array}$ & .27 & $\begin{array}{r}-.04947 \\
(.854)\end{array}$ & $\begin{array}{c}1.17971 \\
(226.187)\end{array}$ & .10 & $\begin{array}{l}.06620 \\
(.190)\end{array}$ & $\begin{array}{l}1.78224 \\
(39.325)\end{array}$ & .34 & $\begin{array}{l}-40486 \\
(30.325)\end{array}$ & $\begin{array}{c}1.62631 \\
(295.644)\end{array}$ \\
\hline $\begin{array}{c}.68755 \\
(29.599)\end{array}$ & .35 & $: 01213$ & $\begin{array}{l}1: 08665 \\
(48.732)\end{array}$ & .04 & $\begin{array}{r}-.27989 \\
(.499)\end{array}$ & $\begin{array}{l}1: 24564 \\
(3.536)\end{array}$ & .33 & $\begin{array}{l}-.40649 \\
(9.223)\end{array}$ & $\begin{array}{r}1,36366 \\
(37.116)\end{array}$ \\
\hline .92744 & .25 & $\begin{array}{r}.06606 \\
(1.253)\end{array}$ & $\begin{array}{c}1.28575 \\
(214.787)\end{array}$ & .10 & $\begin{array}{c}.44921 \\
(19.905)\end{array}$ & $\begin{array}{r}1.08457 \\
(52.508)\end{array}$ & .42 & $\begin{array}{l}-.24102 \\
(35.158)\end{array}$ & $\begin{array}{c}1.32153 \\
(478.320)\end{array}$ \\
\hline $\begin{array}{l}.75633 \\
503.727)\end{array}$ & .28 & $\begin{array}{r}.04793 \\
(3.109)\end{array}$ & $\begin{array}{c}1.20666 \\
(782.501)\end{array}$ & .09 & $\begin{array}{c}.63947 \\
(62.628)\end{array}$ & $\begin{array}{c}1.46163 \\
(129.915)\end{array}$ & .50 & $\begin{array}{c}-.24102 \\
(192.335)\end{array}$ & $\begin{array}{c}1.32153 \\
(1972.801)\end{array}$ \\
\hline $\begin{array}{l}.55354 \\
(17.464)\end{array}$ & .09 & $\begin{array}{l}.02309 \\
(.348)\end{array}$ & $\begin{array}{c}.91004 \\
(80.459)\end{array}$. & .03 & $\begin{array}{l}.42576 \\
(9.353)\end{array}$ & $\begin{array}{l}1.55797 \\
(18.624)\end{array}$ & .36 & $\begin{array}{c}-.28132 \\
(105.437)\end{array}$ & $\begin{array}{c}1.36001 \\
(366.462)\end{array}$ \\
\hline $\begin{array}{l}42564 \\
10.801)\end{array}$ & .03 & $\begin{array}{r}.14466 \\
(3.183)\end{array}$ & $\begin{array}{r}.54459 \\
(4.016)\end{array}$ & .04 & $\begin{array}{r}-.11395 \\
(.146)\end{array}$ & $\begin{array}{l}3.01683 \\
(9.127)\end{array}$ & .19 & $\begin{array}{l}-.13768 \\
(7.383)\end{array}$ & $\begin{array}{l}1.22863 \\
(52.335)\end{array}$ \\
\hline $\begin{array}{l}41669 \\
19.953)\end{array}$ & .26 & $\begin{array}{l}.29371 \\
(5.591)\end{array}$ & $\begin{array}{l}1.01518 \\
(33.355)\end{array}$ & .14 & $\begin{array}{r}-.20146 \\
(.298)\end{array}$ & $\begin{array}{l}2.36439 \\
(20.507)\end{array}$ & .47 & $\begin{array}{l}-.08917 \\
(1.516)\end{array}$ & $\begin{array}{c}1.05619 \\
(106.183)\end{array}$ \\
\hline $\begin{array}{l}.82719 \\
(34.801)\end{array}$ & .31 & - & $\begin{array}{l}2.19020 \\
(96.173)\end{array}$ & .12 & $\begin{array}{l}.49073 \\
(7.300)\end{array}$ & $\begin{array}{l}1.58485 \\
(16.653)\end{array}$ & .25 & $\begin{array}{l}-.16350 \\
(7.254)\end{array}$ & $\begin{array}{l}1.06462 \\
(67.270)\end{array}$ \\
\hline $\begin{array}{c}.76478 \\
345.480)\end{array}$ & .24 & $\begin{array}{r}.08614 \\
(5.066)\end{array}$ & $\begin{array}{c}1.13581 \\
(344.832)\end{array}$ & .09 & $\begin{array}{c}.79561 \\
(51.501)\end{array}$ & $\begin{array}{l}1.18444 \\
(44.687)\end{array}$ & .54 & $\begin{array}{c}-.24993 \\
(107.537)\end{array}$ & $\begin{array}{c}1 . \$ 9516 \\
\left(1^{311.955}\right)\end{array}$ \\
\hline $\begin{array}{c}58040 \\
.93,611)\end{array}$ & .10 & $\begin{array}{r}.06099 \\
(1.393)\end{array}$ & $\begin{array}{l}1.00548 \\
(58.785)\end{array}$ & .04 & $\begin{array}{l}.50367 \\
(7.934)\end{array}$ & $\begin{array}{l}1.82049 \\
(16.100)\end{array}$ & .36 & $\begin{array}{l}-.29076 \\
(80.247)\end{array}$ & $\begin{array}{c}1.19759 \\
(211.475)\end{array}$ \\
\hline $\begin{array}{r}.15327 \\
(.978)\end{array}$ & .03 & $\begin{array}{r}.15399 \\
(1: 991)\end{array}$ & $\begin{array}{r}.62226 \\
(2.914)\end{array}$ & .04 & $\begin{array}{r}-.25985 \\
(.445)\end{array}$ & $\begin{array}{l}3.48888 \\
(7.192)\end{array}$ & . 19 & $\frac{-.15222}{(6.495)}$ & $\begin{array}{l}1.15748 \\
(33.648)\end{array}$ \\
\hline $\begin{array}{c}39332 \\
(18,651)\end{array}$ & .32 & $\begin{array}{r}.32695 \\
(8.295)\end{array}$ & $\begin{array}{c}.95311 \\
(34.222)\end{array}$ & .17 & $\begin{array}{c}-.08318 \\
(.050)\end{array}$ & $\begin{array}{l}2.42404 \\
(20.770)\end{array}$ & .51 & $\begin{array}{l}-.10861 \\
(2.583)\end{array}$ & $\begin{array}{c}1.00313 \\
(106.964)\end{array}$ \\
\hline
\end{tabular}

entre paréntesis en la primera columna (estrato) es el número de observaCaracterística del Crecimiento Industrial; Comercio Exterior, vol. 29, núm. 
En el cuadro 16 se presentan elasticidades-gasto estimadas con base en los datos de la encuesta de 1968 para seis conceptos: Autoconsumo Primario; Tradicional I (Alimentos, bebidas y tabaco); Tradicional II (resto de las manufacturas excepto electrodomésticos y vehículos); Moderno (electrodomésticos y vehículos); y Servicios. Los resultados incluidos de este cuadro muestran que - excepto en los tres primeros estratos del sector rural- la elasticidad-gasto del consumo en alimentos (Primario y Tradicional I) es menor que la unidad y, en general, decrece a medida que aumenta el ingreso, y que nos movemos del sector rural, al urbano y al metropolitano.

La tendencia descendente de la elasticidad es congruente con la ley de Engel; sin embargo, resulta difícil aceptar que la elasticidad de estos dos grupos de bienes sea inferior a la unidad en el estrato de 0-1 000 pesos de los sectores urbano y metropolitano que, como se vio en páginas anteriores, se trata de un grupo que aún no había cubierto los requerimientos mínimos de calorías y proteínas. ${ }^{26}$

Por otra parte, destaca el hecho que para el resto de las manufacturas incluidas en Tradicional II, las elasticidades son bastante superiores a la unidad en el estrato de 0-1000 pesos de las familias rurales, urbanas y metropolitanas. Para estas últimas, incluso la elasticidad para Moderno del estrato de 0-1 000 pesos, resulta superior a uno (1.58).

Estos resultados indican que "... aquellas familias que se encuentran en el estrato de 0-1000 pesos ... tenderían a sustituir proporcionalmente bienes básicos (como los alimentos, que componen el rubro más importante de Primario y Tradicional I) por bienes no básicos o 'de lujo' (como son los de consumo duradero), a medida que sus ingresos aumentan. Este hecho resulta sorprendente desde el momento que se parte del supuesto que las familias urbanas más pobres no han cubierto efectivamente sus necesidades básicas; este fenómeno bien puede explicarse mediante la intensidad con que opera el 'efecto demostración'... y la eficacia de la propaganda en los centros urbanos". ${ }^{27}$ Ello implica que la redistribución del ingreso que garantiza un mejor nivel de vida (en términos de nutrición, por ejemplo), deberá ser relativamente mayor en el sector urbano que en el rural, ya que en el primero el mayor poder adquisitivo de las familias pobres lo absorben proporcionalmente más los bienes no básicos, fenómeno que en América Latina se conoce como el caso del "refrigerador vacío".

Para conocer las tendencias en el consumo ante cambios distributivos, para fechas más recientes, se han calculado elasticidades por intervalo ${ }^{28}$

26 Véase cuadro 13.

27 Lustig, N. (1979), op. cit.

${ }^{28} \mathrm{La}$ fórmula es la siguiente:

$e_{i, j, j+1}=\frac{\left(G_{i, j+1}-G_{i, j}\right) /\left(G_{i, j+1}+G_{i, j}\right) / 2}{\left(G_{j+1}-G_{j}\right) /\left(G_{j+1}+G_{j}\right) / 2}$

$e_{i, j, j+1}$ : elasticidad intervalo $j$ y $j+1$, donde $i$ se refiere al bien, y $j$ y $j+1$ a 


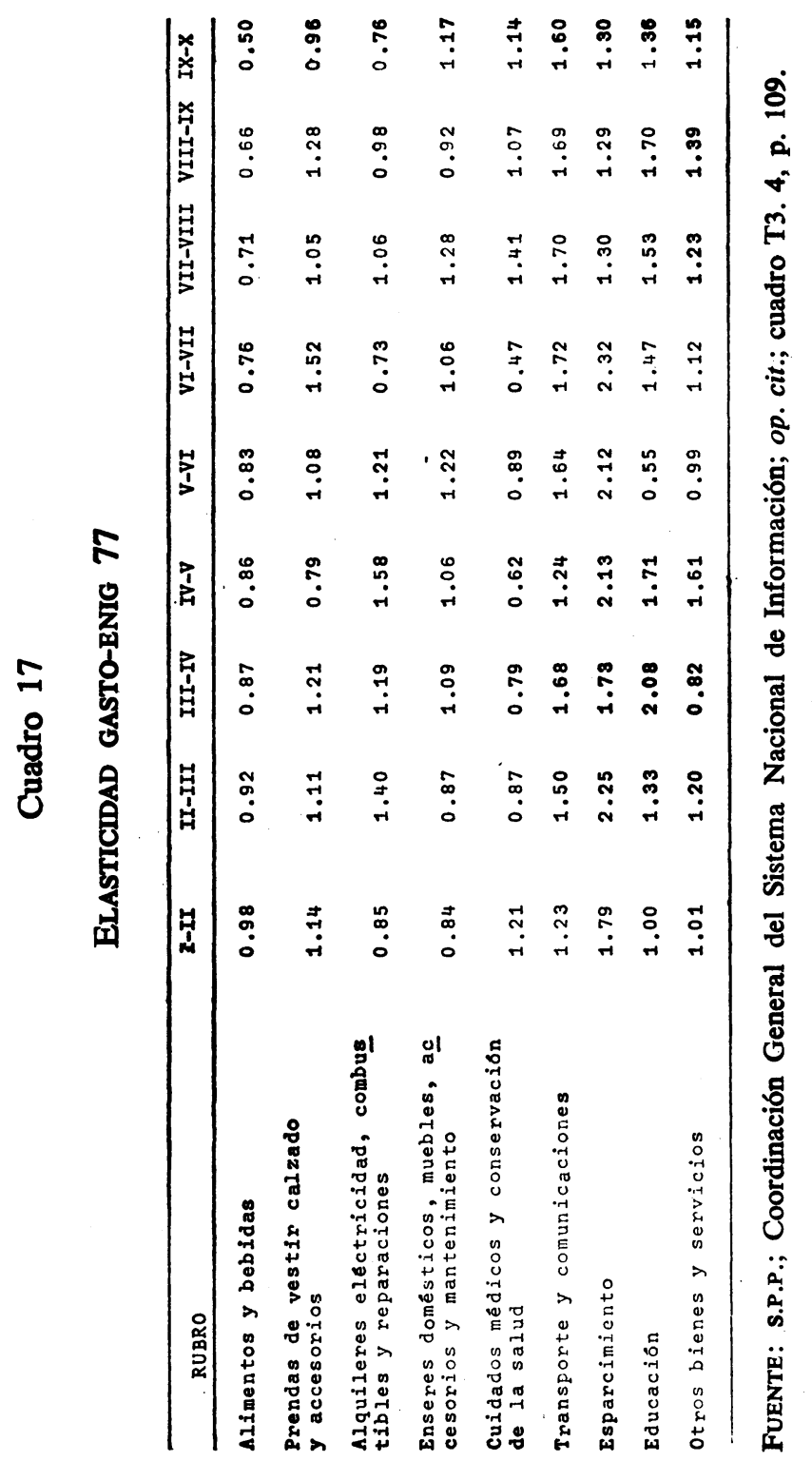


con base en la información disponible para 1977 por rubros y deciles, que se presentan en el cuadro $17 .^{29}$

Los resultados para 1977 son similares a los de 1968 y, por lo tanto, refuerzan las conclusiones anteriores. Por ejemplo, la elasticidad por intervalo para Alimentos y Bebidas de los deciles I al II, del II al III, del III al IV y del IV al V resultan ser inferiores a la unidad: $0.98,0.92,0.87$ y 0.86 , respectivamente; mientras tanto, la que corresponde a Esparcimiento, para los mismos deciles es de $1.79,2.25,1.73$ y 2.13. Aquí como para el caso de 1968, los hogares comprendidos entre el primer y el quinto decil aún no han cubierto los requerimientos mínimos de una alimentación adecuada $^{30} \mathrm{y}$, para garantizar que la mejoren hasta lograr los mínimos necesarios, se requiere de un aumento de los ingresos del grupo más bajo, de tal forma que este grupo dispusiera de ingresos equivalentes al de aquellos que sí logran tener una alimentación adecuada. ${ }^{31}$

\section{Requerimientos redistributivos}

En las páginas anteriores se observó que es a partir del quinto decil que no se observaron deficiencias en el consumo de calorías y proteínas, y que la media del ingreso corriente monetario mensual de este decil fue de 2693 pesos, aproximadamente igual al salario mínimo medio (simple) prevaleciente en 1977 (2 707 pesos). Por lo tanto, se puede considerar que -en términos generales - para lograr una alimentación adecuada, todos los hogares deberían alcanzar por lo menos este nivel de ingreso. ${ }^{32}$

A continuación se presentan los resultados de tres experimentos con el fin de ilustrar sobre las consecuencias e implicaciones de tres situaciones abstractas $y$, hasta cierto punto extremas que pueden servir tanto de señalamiento de futuras tendencias, como de guía para la implementación de políticas.

El primero de estos tres experimentos consiste en estimar el número de años que llevaría a cada uno de los primeros cuatro deciles lograr el umbral de ingreso necesario para lograr una alimentación apropiada ( 2693 pesos mensuales, por hogar), bajo el supuesto de un cierto crecimiento del in-

los deciles; $G_{i, j+1}=$ gasto en el bien $i$, del decil $j+1 ; G_{i, j}=$ gasto en el bien $i$ del decil $j ; \stackrel{G}{j+1}_{j+1}, G_{j}=$ gasto total correspondiente a los deciles $j+1$, $j$, respectivamente.

29 La estimación de elasticidades continuas no es posible si no se usan las observaciones directas.

30 Véase el cuadro 14.

31 Esto es válido, si suponemos que las familias se comportan como lo dice la hipótesis del ingreso absoluto; es decir, que a medida que aumentan sus ingresos adoptan los patrones de consumo de las familias que antes ocupaban ese lugar en la escala.

32 Se dice que es en términos generales porque una mejor alimentación depende no únicamente del nivel global de calorías y proteínas, sino también de su composición. 
greso per capita y sin cambios en la distribución. ${ }^{33}$ En el segundo, se han calculado la magnitud y proporción de una redistribución estática ( $\sin$ crecimiento en el ingreso per capita) de los deciles superiores a los inferiores. Por último, se estimó el número de años que llevaría a cada uno de los primeros cuatro deciles alcanzar el umbral de ingreso de 2693 pesos, bajo el supuesto de que todo el incremento del ingreso global es absorbido por estos cuatro deciles, manteniendo constante el ingreso promedio real del quinto decil en adelante.

Además de suponer una cierta tasa de crecimiento (real) del ingreso per capita, se hicieron dos supuestos fundamentales: constancia de los precios relativos, y de la composición del consumo. Como de costumbre, no se pretende que estos supuestos sean una descripción fidedigna de la realidad, sino que se adoptan con el objeto de aislar la magnitud de ciertos efectos. De hecho, los resultados que se presentan a continuación, se modificarían en dirección negativa si el precio de los alimentos aumentara proporcionalmente más que el de los otros bienes.

\section{Experimento I: Crecimiento sin redistribución}

Aquí se han considerado dos trayectorias de crecimiento del ingreso per capita: una muy optimista, $5 \%$ anual, que se basa en los pronósticos sobre crecimiento del PIB y de la población que se han manejado en varios informes del sector público; la otra, de $3 \%$ anual, basada en la tendencia histórica anterior a la crisis de mediados de los setentas; aunque menor que la primera, ésta es una tasa relativamente alta y corresponde a la que prevaleció en periodos de auge económico.

Los ingresos medios mensuales por hogar en pesos de 1977 correspondientes a los primeros cuatro deciles son: I:406; II:966; III:1 431; y IV:2 005. Si el ingreso per capita creciera durante un largo periodo al $5 \%$ anual, para que el primer decil alcanzara el promedio de ingreso de 2693 pesos, tendrían que pasar casi 39 años; y 21, 13 y 6 años para los deciles II, III y IV, repectivamente. ${ }^{34}$ Bajo la segunda teoría, de $3 \%$ anual, los resultados son: 64 años para el primer decil; 35 para el segundo; 21 para el tercero y 10 para el cuarto.

De estos sencillos experimentos se desprende que, si no se produce ningún cambio en la distribución del ingreso, el primer decil de la población (más de un millón de hogares), por ejemplo, tendría que esperar entre una y casi tres generaciones (dependiendo de la tasa de crecimiento del PIB per capita) para alcanzar un nivel de ingresos que le permita una alimentación apropiada. Esta situación puede volverse más dramática aún si, como se

33 Se hará abstracción de las diferencias en los tamaños de los hogares entre los deciles $\mathrm{y}$, también se supone que la tasa de crecimiento del tamaño promedio del hogar es igual a cero; esto último implica que las tasas de crecimiento del ingreso per capita y por hogar sean iguales.

${ }^{34}$ Este cálculo se realizó usando la fórmula $2693=(1+r)^{t_{x}}$, donde $x$ es la media de ingreso del decil correspondiente y $t$ la incógnita. 
vio en las páginas anteriores, continúan las tendencias de que los deciles más bajos pierdan participación relativa en el ingreso global, y de que la escasez relativa de ciertos alimentos básicos se manifieste de manera cada vez más pronunciada en el alza de sus precios.

\section{Experimento II: Redistribución sin crecimiento}

¿Qué proporción del ingreso debería ser transferida de los deciles superiores para que los primeros cuatro estratos alcancen el umbral de ingreso de $\$ 2693$ ? Si se considera el ingreso del conjunto formado por los deciles VI-X, resulta que habría que transferir un monto equivalente al $15.45 \%$ de éste; la implicación de una redistribución como ésta es que el promedio del grupo baje de 7751 pesos mensuales a 6554 pesos. Si la transferencia se hiciera únicamente desde el último decil, la proporción de ingreso que debiera transferirse es de $35.29 \%$; y la disminución en el ingreso medio del decil sería de 16962 pesos mensuales a 10165 pesos; como puede observarse en el cuadro 14 esta media continuaría siendo la más alta de todas.

Desde luego, una redistribución de este tipo es totalmente utópica y políticamente inaplicable dentro del régimen capitalista; e incluso difícil de poner en práctica aún en un sistema socialista. El objeto de presentarla aquí, como ya se dijo, es totalmente ilustrativo.

Sin embargo, es interesante notar que las modificaciones tanto en los niveles absolutos de ingreso, como en la posición relativa de los deciles que ven disminuidos sus ingresos, no dejarían a estos hogares en situaciones "insostenibles", ya sea en lo que se refiere al consumo de bienes y servicios básicos, o incluso de los "bienes de lujo". ${ }^{35}$ Esto es más cierto aun cuando se considera la subestimación del ingreso de los grupos más ricos, que usualmente traen estas encuestas.

\section{Experimento III: Crecimiento con redistribución}

La hipótesis aquí es que todo el incremento del ingreso per capita es absorbido por los primeros cuatro deciles, mientras permanece constante el ingreso real de los seis restantes. Como se hizo en el primer experimento, también se supondrán dos posibles trayectorias de crecimiento: del $5 \%$ y del $3 \%$ anuales, respectivamente.

Según la encuesta, la media de ingreso global en 1977 fue de 4623 pesos mensuales por hogar; para que los cuatro primeros deciles alcancen el umbral de 2693 pesos sin alterar el ingreso real de los otros, puede demostrarse que es necesario aumentar el ingreso medio global hasta 5222 pesos

35 En 1977, sólo el $9.69 \%$ de las familias percibió un ingreso superior a los 10150 pesos mensuales (véase el cuadro 2). Éste sería aproximadamente el ingreso medio del décimo decil después de la redistribución (10 165 pesos). 
mensuales por hogar. ${ }^{36}$ Bajo la hipótesis de un crecimiento del $5 \%$ anual del ingreso per capita, llevaría 2.5 años lograr la media necesaria; y 4.12 años bajo una tasa de crecimiento del $3 \%$ anual. ${ }^{37}$

Los resultados de este experimento son sumamente significativos porque muestran el grado de rapidez con que se podría mejorar la situación alimentaria del sector en situación crítica, si se toman las medidas necesarias para encauzar los beneficios esperados del crecimiento basado en la explotación petrolera hacia estos grupos de la población. Sin embargo, a pesar de que bajo esta hipótesis los grupos de más altos ingresos no ven dañada su situación absoluta, la viabilidad política de implementar redistribuciones con este grado de intensidad es muy reducida, ya que en la disputa por los "beneficios del petróleo" tendrían una participación muy activa los grupos capitalistas consolidados. Aun cuando la tasa de crecimiento del PIB por habitante logre los niveles esperados, si la situación inflacionaria y la "crisis" de alimentos persisten, las tensiones sociales irán en aumento y será más difícil lograr compromisos políticos con las distintas clases que componen la sociedad mexicana.

En esta situación crítica, la magnitud y rapidez de la mejora del nivel de ingresos de los hogares más pobres de la población (de los primeros cuatro deciles, por ejemplo) dependerá fundamentalmente del número y sobre todo del tipo de empleos que se generen, y de la política que se siga en el sector rural. Esto último es crucial ya que el "modelo" de acumulación que se inicia con la explotación petrolera en gran escala, puede resultar en el beneficio de los grupos medios y medios bajos, pero que al mismo tiempo continúen marginados, malnutridos, abandonados a su suerte, el $20 \%$ inferior de los hogares, alrededor de diez millones de mexicanos.

${ }^{36} \mathrm{El}$ incremento necesario en el ingreso de los primeros cuatro deciles para que éstos logren el nivel de 2693 pesos mensuales, es de 5986 pesos; si se divide esta cifra entre los diez deciles y luego la sumamos al ingreso medio global inicial de 4623.40 pesos, el resultado es 5222 pesos mensuales por hogar.

37 Estas cifras se calcularon con la fórmula $5222=(1+r)-{ }^{t 4} 623.40$. 